\title{
Fórmulas de Substituição para as Integrais de Gauge
}

\section{Márcia Cristina A. Braz Federson}

\author{
DISSERTAÇÃO APRESENTADA \\ $\mathrm{AO}$ \\ INSTITUTO DE MATEMÁTICA E ESTATÍSTICA \\ DA \\ UNIVERSIDADE DE SÃO PAULO \\ PARA OBTENÇÃO DO GRAU \\ DE \\ MESTRE EM MATEMÁTICA
}

Orientador: Prof. Dr. Chaim Samuel Hönig

Área de concentração: Teoria de Integração

-São Paulo, Janeiro de 1993.-

Durante este trabalho, a autora recebeu apoio financeiro do CNPq processos $\mathrm{n}^{\text {os }} 830382.90 / 9,830797.91 / 2,830075.92 / 5$ e da FAPESP procésso ${ }^{\circ}$ 92/1452 4 . 
aos meus avós

Alberto,

Eunice,

Ida e

William. 


\section{AGRADECIMENTOS}

Agradeço, primeiramente, a Deus por ter me conduzido neste trabalho, ao meu marido, Fernando, pelo carinho, apoio e sugestôes e ao Prof. Chaim, pela orientação, paciência e amizade. Aos colegas Leônidas de Oliveira Brandão e Marcelo Messias sou grata pela ajuda com os computadores, e ao Cássio Yukio Shimuta, pelas dicas valiosas. Agradeço também ao Prof. Plínio A. Q. Simões pelo incentivo durante todo o programa de mestrado. 


\section{RESUMO}

Estudamos as integrais vetoriais $\int_{a}^{b} d \alpha(t) f(t)$ e $\int_{a}^{b} \alpha(t) d f(t)$ no sentido de Kurzweil-Henstock para o caso no qual $f:[a, b] \rightarrow X$ e $\alpha:[a, b] \rightarrow L(X, Y)$, onde $[a, b]$ é um intervalo de $\mathbb{R}, X$ e $Y$ são espaços de Banach. Centramos nossa atenção nas propriedades que nos levam a obter as Fórmulas de Substituição para estas integrais:

$$
\int_{a}^{b} d_{t}\left[\int_{a}^{t} d \alpha(s) f(s)\right] g(t)=\int_{a}^{b} d \alpha(t) f(t) g(t),
$$

igualdade que nem sempre é verdadeira, mesmo quando as integrais estão definidas e $\alpha(t)=t$.

\section{ABSTRACT}

We study the vector integrals $\int_{a}^{b} d \alpha(t) f(t)$ and $\int_{a}^{b} \alpha(t) d f(t)$ in the sense of Kurzweil-Henstock for the case in which $f:[a, b] \rightarrow X$ and $\alpha:[a, b] \rightarrow L(X, Y)$, where $[a, b]$ is an interval of $\mathbb{R}, X$ and $Y$ are Banach spaces. We centre our attention to the properties which lead us to obtain the Substitution Formulas for these integrals:

$$
\int_{a}^{b} d_{t}\left[\int_{a}^{t} d \alpha(s) f(s)\right] g(t)=\int_{a}^{b} d \alpha(t) f(t) g(t),
$$

equality not always true, even when the integrals are defined and $\alpha(t)=t$. 


\section{CONTEÚDO}

NOTAÇÃO

INTRODUÇÃO

Capítulo 1: A Integral de Riemann-Stieltjes

1.1 Funções de Variação Limitada

1.2 Funções de Semivariação Limitada e Funções de Variação Fraca Limitada

1.3 A Integral de Riemann-Stieltjes e a Integral Interior

1.4 Integração por partes

Capítulo 2: As Integrais de Gauge

2.1 As Integrais Vetoriais de Kurzweil

2.2 As Integrais Vetoriais de Henstock

2.3 Integrais Vetoriais Impróprias

REFERÊNCIAS

ÍNDICE DE SÍMBOLOS 


\section{NOTAÇÃO}

$X, Y, Z$ e $W$ indicam espaços de Banach, $L(X, Y)$ indica o espaço das funçôes $g: X \rightarrow Y$ lineares e contínuas munido da norma

$$
\|g\|=\sup _{\|x\| \leq 1}\|g(x)\|
$$

e $X^{\prime}$ indica o dual de $X$.

Se $X$ é um espaço de Hilbert e $x, y \in X$, então indicamos o produto interno de $x$ por $y$ por $<x, y>$.

Dado um intervalo compacto $[a, b]$ de $\mathbb{R}$, dizemos que $d: a=t_{0}<t_{1}<\ldots<t_{n}=b$ é uma divisão de $[a, b]$ e indicamos por $D_{[a, b]}$ o conjunto das divisões de $[a, b]$. Se $d_{1}, d_{2} \in D_{[a, b]}$ são tais que $d_{2}$ contém os pontos de $d_{1}$, entâo dizemos que $d_{2}$ é mais fina que $d_{1}$ e pomos $d_{1} \preceq d_{2}$. Para $d: a=t_{0}<t_{1}<\ldots<t_{n}=b$, sejam $|d|=n$ e $\Delta d=\sup \left\{t_{i}-t_{i-1} ; i=1,2, \ldots,|d|\right\}$.

Seja $f:[a, b] \rightarrow X$. Então $D_{f}$ denota o conjunto dos pontos de descontinuidade de $f$. Se $f$ é limitada, escrevemos $\|f\|=\sup _{a \leq t \leq b}\|f(t)\|$. Para cada $\delta>0$,

$$
\omega_{\delta}(f)=\sup \{\|f(t)-f(s)\| ; t, s \in[a, b],|t-s| \leq \delta\}
$$

é a $\delta$-oscilaçào de $f$ em $[a, b]$ e, dada $d \in D_{[a, b]} \operatorname{com} d: a=t_{0}<t_{1}<\ldots<t_{n}=b$, então

$$
\omega_{d}(f)=\sup _{1 \leq i \leq|d|}\left\{\|f(t)-f(s)\| ; t, s \in\left[t_{i-1}, t_{i}\right]\right\}
$$

é a oscilação de $f$ em $[a, b]$ com respeito à $d$, e definimos

$$
\omega_{i}(f)=\sup \left\{\|f(t)-f(s)\| ; t, s \in\left[t_{i-1}, t_{i}\right]\right\} .
$$

Denotamos por $\mathcal{C}\left([a, b], X^{\prime}\right)$ o espaço de Banach das funções contínuas de $[a, b]$ em $X$ munido da seguinte norma:

$$
f \in \mathcal{C}([a, b], X) \mapsto\|f\|=\sup _{a \leq t \leq b}\|f(t)\| \in \mathbb{R}_{+}
$$


Dizemos que $\alpha:[a, b] \rightarrow L(X, Y)$ é uma função fracamente contínua se a função

$$
\alpha \cdot x: t \in[a, b] \mapsto \alpha(t) \cdot x \in Y
$$

é contínua, $\forall x \in X$ e, neste caso, escrevemos $\alpha \in \mathcal{C}^{\sigma}([a, b], L(X, Y))$. Então $\alpha$ é limitada e, com a norma do sup, $\mathcal{C}^{\sigma}([a, b], L(X, Y))$ é um espaço de Banach (pelo teorema de BanachSteinhauss).

Dado $A \subset[a, b]$, definimos a função característica de $A$ por $\chi_{A}(t)=\left\{\begin{array}{l}1, t \in A \\ 0, t \notin A\end{array}\right.$ e denotamos por $\left.f\right|_{A}$ a restrição à. A de uma função $f:[a, b] \rightarrow X$.

Se $\left(x_{n}\right)$ é uma sequência de elementos de $X$ que converge para $x$, então escrevemos $x_{n} \rightarrow x$.

Para $f:[a, b] \rightarrow X$ definimos:

$$
f(t+)=\lim _{h \mid 0} f(t+h), \forall t \in[a, b[
$$

e

$$
\left.\left.f(t-)=\lim _{h\rfloor 0} f(t-h), \forall t \in\right] a, b\right],
$$

quando tais limites existem.

Dizemos que $f:[a, b] \rightarrow X$ é uma função em escada, e pomos $f \in E([a, b], X)$, se existe $d \in D_{[a, b]} \operatorname{com} d: a=t_{0}<t_{1}<\ldots<t_{n}=b$ tal que $\left.f\right|_{] t_{i-1}, t_{i}[}$ é constante, para cada $i \in\{1,2, \ldots, n\}$.

O comprimento de um intervalo $I$ de $\mathbb{R}$ de extremos $u$ e $v$ com $u \leq v$ é dado por $|I|=v-u$. Se $M \subset \mathbb{R}$, entào $\stackrel{\circ}{M}$ e $\bar{M}$ indicam, respectivamente, o interior e o fecho de $M$.

Vamos indicar por $m$ a medida de Lebesgue (e diremos simplesmente medida) e por $\mathcal{L}_{1}(M)$ o espaço das funçôes $f: M \subset \mathbb{R} \rightarrow \overline{\mathbb{R}}$ que sào Lebesgue integráveis indicando por ${ }^{L} \int_{M} f$ a sua integral. $\overline{\mathbb{R}}$ denota a reta extendida $\mathbb{R} \cup\{-\infty,+\infty\}$,

Uma propriedade sobre um conjunto $M \subset \mathbb{R}$ vale quase sempre e escrevemos qs, se o subconjunto de $M$ no qual ela nào vale tem medida zero.

$\operatorname{Em} \mathbb{R}$, a bola de centro $\xi$ e raio $\delta>0$ é denotada por $\left.B_{\delta}(\xi)=\right] \xi-\delta, \xi+\delta[$. 
Indicamos por $l_{p}([a, b])$ o espaço vetorial formado pelas famílias $x=\left(x_{t}\right)_{t \in[a, b]}$ de números complexos tais que $\|x\|_{p}<\infty$, munido da norma $x \in l_{p}([a, b]) \mapsto\|x\|_{p}$, onde

$$
\|x\|_{p}=\left(\sum_{t \in[a, b]}\left|x_{t}\right|^{p}\right)^{\frac{1}{p}}
$$

quando $1 \leq p<\infty$ e

$$
\|x\|_{\infty}=\sup _{t \in[a, b]}\left|x_{t}\right|
$$

O espaço vetorial da classe de equivalência das funções mensuráveis $f:[a, b] \rightarrow \mathbb{R}$ tais que $\|f\|_{p}<\infty$, onde

$$
\|f\|_{p}=\left[L \int_{a}^{b}|f()|^{p}\right]^{\frac{1}{p}}
$$

quando $1 \leq p<\infty$ e

$$
\|f\|_{\infty}=\inf \{c ;|f()| \leq c q s\}
$$

é indicado por $L_{p}([a, b])$ e $f \in L_{p}([a, b]) \mapsto\|f\|_{p}$ é a sua norma.

Quando definirmos um termo qualquer, vamos escrevê-lo em negrito.

Usamos o símbolo $\square$ para indicar o término de uma demonstração.

Outros símbolos e notaçòes podem ser encontrados no ÍNDICE DE SÍMBOLOS. 


\section{INTRODUÇÃO}

Uma das nossas primeiras questões em Matemática e que envolve Teoria de Integração é a necessidade de calcularmos a área sob o gráfico de uma função. A idéia mais utilizada para aproximação do valor desta área é através da soma das áreas de retângulos.

$\mathrm{Na}$ aproximação da integral de Riemann por somas de Riemann, exige-se apenas que a máxima amplitude do comprimento dos intervalos seja menor do que um certo $\delta$. Logo, uma divisão de $[a, b]$ não considera qualquer propriedade especial que a função possa ter. Além disso, o espaço das funções Riemann integráveis é muito pequeno, portanto não tem boas propriedades de convergência.

A integral de Lebesgue amplia a integral de Riemann através de uma definição completamente diferente. Contudo, as funções Lebesgue integráveis não contém as integrais de Riemann impróprias nem as suas integrais impróprias.

Denjoy e Perron (1912-1914) deram uma definição que abrange a integral de Lebesgue e as integrais impróprias. Mais tarde (1957-1961), Kurzweil e Henstock conseguiram dar uma definição riemaniana para a integral de Denjoy-Perron.

A idéia das integrais de Kurzweil e de Henstock é tomar os intervalos $I_{i}$ de uma divisão de $[a, b], \operatorname{com} x_{i} \in I_{i}$, tais que o comprimento de cada $I_{i}$ dependa do comportamento da função perto de $x_{i}$. Daí a noção de gauge: uma funçào que a cada ponto de $[a, b]$ associa um número estritamente positivo. Este número vai determinar o comprimento máximo de cada um dos subintervalos de uma divisão de $[a, b]$.

Se ainda, na definiçào de integral de Kurzweil, não fizermos a exigência de que $x_{i} \in I_{i}$, onde os $I_{i}$ sào subintervalos de una divisào de $[a, b]$, então temos a integral de McShane. Esta mudança aparentemente insignificante empobrece este subconjunto do espaço das funções Kurzweil integráveis no sentido de que a integral de McShane não mais contém as integrais 
impróprias. Por outro lado, o espaço das funções McShane integráveis é equivalente ao espaço das funções Lebesgue integráveis (veja [9]) e, no caso de funções a valores num espaço de Banach, o espaço das funçôes McShane-Henstock integráveis é equivalente ao espaço das funções Bochner-Lebesgue integráveis (veja [7]). Estes fatos tornam mais fácil o manuseio de certas integrais devido à simplicidade da definição de McShane.

Este trabalho traz a extensão das integrais de Kurzweil e de Henstock para funções de variação limitada e mais gerais, num contexto de espaços vetoriais, e está centrado em demonstrar a. Fórmula de Substituição (veja [3])

$$
\int_{a}^{b} d_{t}\left[\int_{a}^{t} d \alpha(s) f(s)\right] g(t)=\int_{a}^{b} d \alpha(t) f(t) g(t)
$$

que não é nem verdadeira no caso particular em que $\alpha(t)=t$ para funções à valores em um espaço de Banach. Senão vejamos. Sejam $Y=\mathbb{R}, X=l_{2}([a, b]), g:[a, b] \rightarrow X$ dada por $g(t)=\epsilon_{t}$ onde $e_{t}(s)=1$, se $s=t$ e $e_{t}(s)=0$, se $s \neq t$ e $f:[a, b] \rightarrow X^{\prime}$ dada por $f(t)=\tilde{e}_{t}$ onde $\check{e}_{t}(x)=<\epsilon_{t}, x>, \forall x \in X^{\prime}$. Então $f(t) g(t)=<e_{t}, e_{t}>=1 \mathrm{e}$, portanto,

$$
\int_{a}^{b} f(t) g(t) d t=\int_{a}^{b} d t=b-a .
$$

Por outro lado,

$$
\int_{a}^{b} f(t) d t=0
$$

o que implica que

$$
\int_{a}^{b} d_{t}\left[\int_{a}^{t} f(s) d s\right] g(t)=0
$$

Logo, para $b>a$ temos

$$
0<b-a=\int_{a}^{b} f(t) g(t) d t \neq \int_{a}^{b} d_{t}\left[\int_{a}^{t} f(s) d s\right] g(t)=0 .
$$

Para maiores detalhes veja também o Exemplo 3 da página 37.

Obs.: Excetuado o fato de as integrais de McShane não conterem as integrais impróprias, conforme já comentamos, todos os resultados aqui obtidos podem ser aplicados de modo análogo para estas integrais, ou seja, os resultados válidos para as integrais vetoriais de Kurzweil (Henstock) ainda sâo verdadeiros para as integrais vetoriais de McShane (McShaneHenstock) feitas as respectivas alteraçòes nas hipóteses. 
No Capítulo 1, estabelecemos conceitos que são utilizados ao longo do trabalho. São eles função de variação limitada (1.1), função de semivariação limitada e função de variação fraca limitada (1.2), além de outras definições. Aproveitamos também este capítulo para estudar as fórmulas de substituiçâo para as integrais de Riemann-Stieltjes (1.3 e 1.4) (caso particular das integrais de gauge quando o gauge é uma função constante).

Dividimos o Capítulo 2 em três parágrafos. Os dois primeiros tratam das integrais vetoriais de Kurzweil e de Henstock respectivamente. Os últimos resultados de cada um destes parágrafos focalizam as fórmulas de substituição para estas integrais. O parágrafo 2.3 é dedicado às integrais de gauge impróprias. 


\section{Capítulo 1}

\section{A Integral de Riemann-Stieltjes}

\subsection{Funções de Variação Limitada}

O objetivo deste parágrafo é definir funçào de variaçào limitada e dar as propriedades principais do espaço destas funçòes. O último resultado deste parágrafo é um interessante teorema de decomposição de funções de variação limitada a valores reais, conseqüência de propriedades particulares destas funções. 
Definição 1.1.1 Consideremos a função $f:[a, b] \rightarrow X$.

(i) Se $d \in D_{[a, b]}$ com $d: a=t_{0}<t_{1}<\ldots<t_{n}=b$, então definimos a d-variação de $f$ em $[a, b]$ por:

$$
V_{d}(f)=\sum_{i=1}^{n}\left\|f\left(t_{i}\right)-f\left(t_{i-1}\right)\right\|
$$

(ii) Se $\sup _{d \in D_{[a, b]}} V_{d}(f)<\infty$, então dizemos que $f$ é de variação limitada em $[a, b] e$ escrevemos $f \in B V([a, b], X)$. Definimos a variação de $f$ em $[a, b]$ por:

$$
V(f)=V_{[a, b]}(f)=\sup _{d \in D_{[a, b]}} V_{d}(f) .
$$

É imediato que

Proposição 1.1.2 Se $f \in B V([a, b], X)$ temos:

(i) se $c \in] a, b\left[\right.$, então $V_{[a, b]}(f)=V_{[a, c]}(f)+V_{[c, b]}(f)$;

(ii) a funçâo $t \in[a, b] \mapsto V_{[a, t]}(f) \in \mathbb{R}_{+}$é crescente;

(iii) $f$ é limitada e $\|f(t)\| \leq\|f(a)\|+V_{[a, t]}(f), \forall t \in[a, b]$.

Se $X=\mathbb{R}$, entào $B V([a, b], \mathbb{R})$ é um espaço de funções deriváveis qs, o qual vamos indicar simplesmente por $B V([a . b])$. Este fato segue do Lema 1.1.3 e do Teorema 1.1.4 a seguir.

Lema 1.1.3 Se $f:[a, b] \rightarrow \mathbb{R}$ é crescente, então $f$ é derivável qs e a derivada $f^{\prime}$ é tal que $f^{\prime} \in \mathcal{L}_{1}([a, b]) \operatorname{com} L \int_{a}^{b} f \leq f(b)-f(a)$.

Prova (veja [11], pág. 100, teor. 3). 
Teorema 1.1.4 Seja $f \in B V([a, b])$. Então temos que

(i) existem funções crescentes $f_{1}, f_{2}:[a, b] \rightarrow \mathbb{R}$ tais que $f=f_{1}-f_{2}$;

(ii) $f$ é derivável qs e a derivada $f^{\prime}$ é tal que $f^{\prime} \in \mathcal{L}_{1}([a, b])$.

Prova (para (i), basta tomarmos $f_{1}(t)=V_{[a, t]}(f)$ e $f_{2}(t)=V_{[a, t]}(f)-f(t) \forall t \in[a, b]$ e (ii) segue de (i) e do lema anterior).

Lema 1.1.5 Seja $f \in B V([a, b], X)$. Definindo a função $v_{f}(t)=V_{[a, t]}(f)$, para $t \in[a, b]$, temos:

(i) $v_{f}(t+)-v_{f}(t)=\|f(t+)-f(t)\|, \forall t \in[a, b[$;

(ii) $\left.\left.v_{f}(t)-v_{f}(t-)=\|f(t)-f(t-)\|, \forall t \in\right] a, b\right]$.

Prova (veja [2], pág. 29, prop. 2.8).

Teorema 1.1.6 Se $f \in B V([a, b], X)$, então o conjunto dos pontos de descontinuidade de $f$ é enumerável.

Prova (veja [2], pág. 30, prop. 2.10).

O exemplo a seguir mostra que mesmo uma funçào numérica contínua pode ter variação infinita e, portanto, nào ser de $B V([a, b])$.

Exemplo 1 Consideramos $f:[0,1] \rightarrow \mathbb{R}$ dada por

$$
f(t)=\left\{\begin{array}{l}
\left.\left.t \sin \left(\frac{1}{t}\right) ; t \in\right] 0,1\right] \\
0: t=0
\end{array} .\right.
$$

Agora, vamos definir um espaço de funçòes que contém propriamente o espaço das funçôes de variaçào limitada. Tal espaço é o espaço das funçòes chamadas regradas. 
Definição 1.1.7 Podemos definir:

(i) $f:[a, b] \rightarrow X$ é uma função regrada, e escrevemos $f \in G([a, b], X)$, se $\exists f(t+), \forall t \in[a, b[$

$e$

$$
\exists f(t-), \forall t \in] a, b] .
$$

(ii) $\alpha:[a, b] \rightarrow L(X, Y)$ é uma função fracamente regrada se a função

$$
\alpha \cdot x: t \in[a, b] \mapsto \alpha(t) \cdot x \in Y
$$

é regrada, $\forall x \in X$. Neste caso, escrevemos $\alpha \in G^{\sigma}([a, b], L(X, Y))$ com

$$
\alpha(t \dot{+}) \cdot x=(\alpha \cdot x)(t+) \text { e } \alpha(t \dot{-}) \cdot x=(\alpha \cdot x)(t-) .
$$

Obs.: usando o de Banach-Steinhauss, demonstra-se que $\alpha(t \dot{+})$ e $\alpha(t \dot{-})$ existem e pertencem à $L(X, Y)$.

Com a norma do sup. lanto $G([a, b], X)$ como $G^{\sigma}([a, b], L(X, Y))$ são espaços de Banach e as funçôs pertencentes à estes espaços sào limitadas.

Então, pode-se mostrar que o espaço das funçôes em escada é denso no espaço das funçôes regradas.

Proposição 1.1.8 Seja $f:[a, b] \rightarrow \mathbb{X}$. Súo equivalentes as seguintes afirmações:

(i) $f \in G([a, b], X)$ :

(ii) $\forall \varepsilon>0, \exists f_{\varepsilon} \in E\left([a, b, X)\right.$ tal que $\forall t \in[a, b]$ tem-se que $\left\|f(t)-f_{\varepsilon}(t)\right\|<\varepsilon$;

(iii) $\forall \varepsilon>0, \exists d: a=t_{0}<t_{1}<\ldots<t_{n}=b$ tal que $\forall i \in\{1,2, \ldots, n\}$, se $\left.t, s \in\right] t_{i-1}, t_{i}[$, entĩo $\|f(t)-f(s)\|<\varepsilon$.

Prova (veja [4]. teor. 3.1). 
Também pode-se mostrar que

Teorema 1.1.9 $B V([a, b], X) \subset G([a, b], X)$.

Prova (veja [2], pág. 28, teor. 2.7).

Agora, vamos definir função absolutamente contínua para, em seguida, darmos a versão do Teorema Fundamental do Cálculo para as integrais de Lebesgue: o Teorema de Lebesgue.

Depois, definimos função singular para, então, chegarmos ao teorema de decomposição da uma funçào de $B V([a, b])$ como soma de uma função absolutamente contínua, uma função singular e uma componente de salto.

Definição 1.1.10 Dizemos que $f:[a, b] \rightarrow \mathcal{X}$ é absolutamente contínua em $[a, b]$ se: $\forall \varepsilon>0, \exists \delta>0$ tal que $\sum_{i=1}^{n}\left\|f\left(y_{i}\right)-f\left(x_{i}\right)\right\|<\varepsilon$, para toda família finita $\left\{I_{1}, I_{2}, \ldots, I_{n}\right\}$ de intervalos interiormente disjuntos, com $\left.\stackrel{\circ}{I}_{i}=\right] x_{i}, y_{i}\left[\right.$ para cada $i$, e tal que $\sum_{i=1}^{n}\left|I_{i}\right|<\delta$. Neste caso, escrevemos $f \in A C([a, b], X)$ e, quando $X=\mathbb{R}$, escrevemos $f \in A C([a, b])$.

Daí, segue da definiçào que

Teorema 1.1.11 $A C^{\prime}([a, b], X) \subset B Y^{\prime}\left([a, b], X^{*}\right)$

\section{Teorema 1.1.12 (TEOREMA DE LEBESGUE)}

(i) Seja $F \in A C([a, b])$. Entào $F^{\prime} \in \mathcal{L}_{1}([a, b]) \in L \int_{a}^{\xi} F^{\prime}=F(\xi)-F(a), \forall \xi \in[a, b]$.

(ii) Sejam $f \in \mathcal{L}_{1}([a, b]) \in F:[a, b] \rightarrow \mathbb{R}$ definida por $F(\xi)=L \int_{a}^{\xi} f$. Entâo temos que $F \in A C^{\prime}([a, b]) \in F^{\prime}=f q s$. 
Prova (veja [11], pág. 110, teor. 14).

É bem conhecido o exemplo da função contínua monótona crescente de $[0,1]$ sobre $[0,1]$ que é constante fora do conjunto triádico de Cantor. Para esta função temos $f^{\prime}=0 q s$ e $f \notin A C([0,1])$, apesar de $f$ ser de variaçào limitada.

Definição 1.1.13 Seja $f \in \mathcal{C}([a, b], R) \cap B V([a, b])$. Então $f$ é singular se e somente se $f^{\prime}=0$ qs. Neste caso, escrevemos $f \in \mathcal{C}_{\text {sing }}([a, b])$.

Lema 1.1.14 Seja $f \in \mathcal{L}_{1}(E)$. Entiò. $\forall \varepsilon>0, \exists \delta>0$ tal que $\forall A \subset E \operatorname{com} m(A)<\delta$ tem-se que $L \int_{A}|f|<\varepsilon$.

Prova (basta tomarmos $f=f^{+}-f^{-}$e aplicarmos a prop. 14, pág. 88 de [11]).

Teorema 1.1.15 Seja $f \in B V([a, b])$. Então existe uma decomposição de $f$ como soma de uma funçâo de sallo. uma funçào singular e uma funçâo absolutamente contínua.

\section{Prova}

Por hipótese, $f \in B V([a, b])$, entào $f$ é contínua exceto em uma quantidade enumerável de pontos (Teorema 1.1.6).

Para cada $\xi \in D_{f} \cap\left[a, b\left[\right.\right.$, seja $c_{\xi+}=f(\xi+)-f(\xi)$ e, para cada $\left.\left.\xi \in D_{f} \cap\right] a, b\right]$, seja $c_{\xi-}=f(\xi)-f(\xi-)$.

Portanto, para cada $\xi \in D_{f} \cap\left[a, b\left[\right.\right.$, a funçào $f-c_{\xi+} \cdot \chi \chi_{\xi, b]}$ é contínua à direita em $\xi$, pois

$$
\left.\left.\left.\left[f(\xi+)-c_{\xi+} \cdot \backslash\right] \xi, b\right](\xi+)\right]-\left[f(\xi)-c_{\xi+} \cdot \chi_{]} \xi, b\right](\xi)\right]=0
$$


e, do mesmo modo, para cada $\left.\left.\xi \in D_{f} \cap\right] a, b\right]$, a função $f-c_{\xi-} \cdot \chi_{[\xi, b]}$ é contínua à esquerda em $\xi$, pois

$$
\left[f(\xi)-c_{\xi-} \cdot \chi_{[\xi, b]}(\xi)\right]-\left[f(\xi-)-c_{\xi-} \cdot \chi_{[\xi, b]}(\xi-)\right]=0
$$

Consideremos a funçãoo $v_{f}:[a, b] \rightarrow \mathbb{R}$ dada por $v_{f}(t)=V_{[a, t]}(f)$.

Como $v_{f}$ é crescente (Proposição 1.1.2), então

$$
\sum_{t \in D_{f} \cap[a, b[}\left[v_{f}(t+)-v_{f}(t)\right]+\sum_{\left.\left.t \in D_{f} \cap\right] a, b\right]}\left[v_{f}(t)-v_{f}(t-)\right] \leq V(f) .
$$

Daí, segue que as séries $\sum_{t \in D_{f} \cap[a, b[}\|f(t+)-f(t)\|$ e $\sum_{\left.\left.t \in D_{f} \cap\right] a, b\right]}\|f(t)-f(t-)\|$ são absolutamente convergentes (veja o Lema 1.1 .5 ) e, então, a função de salto $f_{1}:[a, b] \rightarrow \mathbb{R}$ definida por

$$
\left.\left.f_{1}=\sum_{\xi \in D_{f} \cap[a, b[} c_{\xi+} \cdot \lambda\right] \xi, b\right]+\sum_{\left.\left.\xi \in D_{f} \cap\right] a, b\right]} c_{\xi-} \cdot \chi[\xi, b]
$$

é tal que $f_{1} \in B V([a, b])$.

Além disso, $f-f_{1}$ é contínua, pois $f_{1}(\xi+)-f_{1}(\xi)=f(\xi+)-f(\xi), \forall \xi \in\left[a, b\left[\right.\right.$ e $f_{1}(\xi)-$ $\left.\left.f_{1}(\xi-)=f(\xi)-f(\xi-), \forall \xi \in\right] a, b\right]$.

E, $f, f_{1} \in B V([a, b])$ implica que $f-f_{1} \in B V([a, b])$.

Então, fazendo $h=f-f_{1}$, temos que $h \in B V([a, b])$ e é contínua.

Daí, pelo Teorema 1.1 .4 (ii) temos que $\exists h^{\prime}$ qs e $h^{\prime} \in \mathcal{L}_{1}([a, b])$.

Logo,

$$
\exists f_{2}(t)=L \int_{a}^{t} h^{\prime}, \forall t \in[a, b] .
$$

Então, pelo Lema 1.1.14, $f_{2} \in A C([a, b])$.

Agora, colocando $f_{3}=h-f_{2}$, temos que $f_{3} \in B V([a, b])$ e isto implica que $\exists f_{3}^{\prime} q s$, pelo Teorema 1.1.4 (ii). 
Assim,

$$
L \int_{a}^{t} f_{3}^{\prime}=L \int_{a}^{t} h^{\prime}-L \int_{a}^{t} f_{2}^{\prime}=f_{2}(t)-\left[f_{2}(t)-f_{2}(a)\right]=f_{2}(a), \forall t \in[a, b],
$$

pela definição de $f_{2}$ e pelo Teorema de Lebesgue (Teorema 1.1.12 (i)).

Logo, $f_{3}^{\prime}=0 q s$, isto é, $f_{3}$ é singular.

Entào $f=f_{1}+f_{2}+f_{3} \operatorname{com} f_{1}$ uma função de salto, $f_{2} \in A C([a, b])$ e $f_{3} \in \mathcal{C}_{\text {sing }}([a, b])$. 


\subsection{Funções de Semivariação Limitada e Funções de Variação Fraca Limitada}

As funçòes de semivariação limitada e as funções de variação fraca limitada, que passamos a definir, são funções mais gerais do que as funções de variação limitada estudadas no parágrafo anterior. Vejamos:

Definição 1.2.1 Seja a : $[a, b] \rightarrow L(\mathbb{X}, Y)$. Então:

(i) dada d: $a=t_{0}<t_{1}<\ldots<t_{n}=b$, a d-semivariação de a em $[a, b]$ é dada por:

$$
S V_{d}(\alpha)=\sup \left\{\left\|\sum_{i=1}^{n}\left[\alpha\left(t_{i}\right)-\alpha\left(t_{i-1}\right)\right] \cdot x_{i}\right\| ; x_{i} \in X \quad \text { e }\left\|x_{i}\right\| \leq 1\right\}
$$

(ii) a semivariaçào de $\alpha$ em $[a, b]$ é dada por:

$$
S V(\alpha)=S V_{[a, b]}(\alpha)=\sup _{d \in D_{[a, b]}} S V_{d}(\alpha)
$$

e, se SV $(\alpha)<\infty$. entaio dizemos que $\alpha$ é de semivariação limitada e escrevemos $\alpha \in S V([a, b], L(\mathcal{X}, Y))$;

(iii) se $\mathrm{X}=\mathbb{R}$. cntũo escrevemos $W^{\prime}(\alpha)$ em vez de $S V(\alpha)$ e $B W([a, b], Y)$ em vez de $S V([a, b], L(\mathbb{R}, Y))$. Chamamos $W(a)$ de variação fraca de $\alpha \in B W([a, b], Y)$ de espaço das funções de variação fraca limitada. Obviamente,

$$
W_{d}(a)=\sup \left\{\left\|\sum_{i=1}^{|d|} \lambda_{i}\left[\alpha\left(t_{i}\right)-\alpha\left(t_{i-1}\right)\right]\right\| ; \lambda_{i} \in \mathbb{R} e\left|\lambda_{i}\right| \leq 1\right\}
$$

$\epsilon$

$$
W(\alpha)=W_{[a, b]}(\alpha)=\sup _{d \in D_{[a, b]}} W_{d}(\alpha)
$$

Entào. da definiçào segue que 
Proposição 1.2.2 $B V([a, b], L(X, Y)) \subset S V([a, b], L(X, Y)) \subset B W([a, b], L(X, Y))$.

Damos a seguir um exemplo muito simples de uma função de $B W([a, b], X)$ que não está em $B V([a, b], X)$.

Exemplo 2 Consideremos $X=L_{\infty}([a, b])$.

Seja $f:[a, b] \rightarrow L_{\infty}([a, b])$ dada por $f(t)=\chi_{[a, t]}$.

Entâo, para $d \in D_{[a, b]}$ com $d: a=t_{0}<t_{1}<\ldots<t_{n}=b$ temos

$$
\left\|f\left(t_{i}\right)-f\left(t_{i-1}\right)\right\|_{\infty}=\left\|\chi \chi_{\left.t_{i-1}, t_{i}\right]}\right\|_{\infty}=1, \forall i .
$$

Portanto, $V_{d}(f)=|d|$ o que implica que $V(f)=\infty$ e $f \notin B V([a, b], X)$.

Porém,

$$
\left.\left.W_{d}(f)=\left\|\sum_{i=1}^{n} \lambda_{i}\left[f\left(t_{i}\right)-f\left(t_{i-1}\right)\right]\right\|_{\infty}=\| \sum_{i=1}^{n} \lambda_{i} \cdot \chi\right] t_{1-1}, t_{i}\right] \|_{\infty} \leq 1,
$$

$\operatorname{para} \lambda_{i} \in \mathbb{R} \operatorname{com}\left|\lambda_{i}\right| \leq 1 . \forall i$.

Dai, $W(f)=1$ e $f \in B W([a, b], X)$.

Mais geralmente temos que

Proposição 1.2.3 $B \mathrm{~V}([a, b], \mathrm{X})=B W([a, b], \mathrm{X})$ se e só se a dimensão de $X$ é finita.

Prova (veja [10])

É imediato que 
Proposição 1.2.4 Temos

(i) se $\alpha \in B W([a, b], X)$, então $\alpha$ é limitada $e$

$$
\|\alpha(t)\| \leq\|\alpha(a)\|+B W(\alpha), \forall t \in[a, b] .
$$

(ii) se a $\in S V([a, b], L(X, Y))$, entâo $\alpha$ é limitada e

$$
\|\alpha(t)\| \leq\|\alpha(a)\|+S V(\alpha), \forall t \in[a, b] .
$$




\subsection{A Integral de Riemann-Stieltjes e a Integral Interior}

Neste parágrafo, definimos integral de Riemann-Stieltjes e integral interior e damos alguns teoremas sobre a existência da integral de Riemann-Stieltjes. Depois, definimos e damos propriedades das funçòes integrais de funçòes Riemann-Stieltjes integráveis.

Definição 1.3.1 Seja $d \in D_{[a, b]} \operatorname{com} d: a=t_{0}<t_{1}<\ldots<t_{n}=b$.

(i) O conjunto $D=\left\{\left(\xi_{i}, t_{i}\right) ; i=1,2, \ldots, n\right\}$, onde $\xi_{i} \in\left[t_{i-1}, t_{i}\right]$ para cada $i$, é chamado uma divisão marcada de $[a, b]$;

(ii) O conjunto $D^{\bullet}=\left\{\left(\xi_{i}, t_{i}\right) ; i=1,2, \ldots, n\right\}$, onde $\left.\xi_{i} \in\right] t_{i-1}, t_{i}[$ para cada $i$, é chamado uma divisão interior marcada de $[a, b]$.

Denotamos por $T D_{[a, b]}$ o conjunto das divisóes marcadas de $[a, b]$ e por $T D_{[a, b]}^{\bullet}$ o conjunto das divisóes interiores marcadas de $[a, b]$.

Sejam $f:[a, b] \rightarrow X$ e $\alpha:[a, b] \rightarrow L(X, Y)$. Se $D=\left\{\left(\xi_{i}, t_{i}\right) ; i=1,2, \ldots, n\right\} \in T D_{[a, b]}$, entào $|D|=n, \Delta D=\sup \left\{t_{i}-t_{i-1} ; i=1,2, \ldots,|D|\right\}, s^{\alpha}(f, D)=\sum_{i=1}^{|D|}\left[\alpha\left(t_{i}\right)-\alpha\left(t_{i-1}\right)\right] f\left(\xi_{i}\right)$ e $s^{f}(\alpha, D)=\sum_{i=1}^{|D|} \alpha\left(\xi_{i}\right)\left[f\left(t_{i}\right)-f\left(t_{i-1}\right)\right]$. Quando nào for preciso explicitar $|D|$, escrevemos simplesmente $D=\left(\xi_{i}, t_{i}\right)$ para uma divisào marcada de $[a, b]$.

Definição 1.3.2 Dadas $D=\left\{\left(\xi_{i}, t_{i}\right) ; i=1,2, \ldots, n\right\}$ e $D^{\prime}=\left\{\left(\xi_{j}^{\prime}, t_{j}^{\prime}\right) ; j=1,2, \ldots, m\right\}$ em $T D_{[a, b]}$, dizemos que $D$ é mais fina que $D^{\prime}$. e pomos $D^{\prime} \preceq D$. se $d$. $d^{\prime} \in D_{[a, b]}$, onde $d: a=t_{0}<t_{1}<\ldots<t_{n}=b \in d^{\prime}: a=t_{0}^{\prime}<t_{1}^{\prime}<\ldots<t_{m}^{\prime}=b$. são tais que $d^{\prime} \preceq d$. 
Definição 1.3.3 Sejam $f:[a, b] \rightarrow X$ e $\alpha:[a, b] \rightarrow L(X, Y)$. Para $D \in T D_{[a, b]}$ definimos as integrais de Riemann-Stieltjes por:

(i)

$$
\int_{a}^{b} d \alpha(t) f(t)=\lim _{\Delta D \rightarrow 0} s^{\alpha}(f, D)
$$

quando tal limite existe $e$, neste caso, escrevemos $f \in R^{\alpha}([a, b], X)$ (se $\alpha(t)=t$, escrevemos $\left.f \in R([a . b], X) \in \int_{a}^{b} d \alpha(t) f(t)=\int_{a}^{b} f(t) d t\right)$;

(ii)

$$
\int_{a}^{b} \alpha(t) d f(t)=\lim _{\Delta D \rightarrow 0} s^{f}(\alpha, D)
$$

quando tal limite existe $e$, neste caso, escrevemos $\alpha \in R^{f}([a, b], L(X, Y))$.

Usando divisòes marcadas interiores, podemos definir integrais mais gerais do que as integrais de Riemann-Stielt.jes. Sào elas as integrais interiores.

Definição 1.3.4 Sejam $f:[a, b] \rightarrow X \in a:[a, b] \rightarrow L(X, Y)$. Definimos as integrais interiores por:

(i)

$$
\int_{a}^{b} \cdot d \alpha(t) f(t)=\lim _{D \bullet \in T D_{[a, b]}^{\bullet}} s^{\alpha}\left(f, D^{\bullet}\right)
$$

quando tal limite existe $\epsilon$. neste caso, escrevemos $f \in I^{\alpha}([a, b], X)$;

(ii)

$$
\int_{a}^{b} \cdot \alpha(t) d f(t)=\lim _{D \cdot \in T D_{[a, b]}^{\bullet}} s^{f}\left(\alpha, D^{\bullet}\right)
$$

quando tal limite existe $\epsilon$, neste caso. escrevemos $\alpha \in I^{f}([a, b], L(X, Y))$.

Obs.: Dizer que $\exists \lim _{D^{\bullet} \in T D_{[a, \ell]}^{\bullet}} s^{\circ}\left(f . D^{\bullet}\right)$ é equivalente à dizer que existe $I \in Y$ satisfazendo:

$$
\forall \Xi>0 . \exists D_{\varepsilon}^{\bullet} \in T D_{[a, b]}^{\bullet} \text { lal que } \forall D^{\bullet} \supset D_{\varepsilon}^{\bullet} \text { temos que }\left\|I-s^{\alpha}\left(f, D^{\bullet}\right)\right\|<\varepsilon \text {. }
$$

No que segue vamos assumir $a:[a, b] \rightarrow L(\mathrm{X} . \mathrm{Y})$ e $f:[a, b] \rightarrow \mathrm{X}$. 
Proposição 1.3.5 Temos:

(i) $R^{\alpha}([a, b], X) \subset I^{\alpha}([a, b], X)$ e, para $f \in R^{\alpha}([a, b], X)$ temos

$$
\int_{a}^{b} d \alpha(t) f(t)=\int_{a}^{b} \cdot d \alpha(t) f(t)
$$

(ii) $R^{f}([a, b], L(X, Y)) \subset I^{f}([a, b], X) e$, para $\alpha \in R^{f}([a, b], L(X, Y))$ temos

$$
\int_{a}^{b} \alpha(t) d f(t)=\int_{a}^{b} \cdot \alpha(t) d f(t)
$$

Prova (observemos que $T D_{[a, b]}^{\bullet} \subset T D_{[a, b]}$, que $\exists \lim _{\Delta d \rightarrow 0} s^{\alpha}(f, D)$ implica que $\exists \lim _{D^{\bullet} \in T D_{[a, b]}^{\bullet}} s^{\alpha}\left(f, D^{\bullet}\right)$ e que $\exists \lim _{\Delta d \rightarrow 0} s^{f}(\alpha, D)$ implica que $\left.\exists \lim _{D^{\bullet} \in T D_{[a, b]}^{\circ}} s^{f}\left(\alpha, D^{\bullet}\right)\right)$.

Entretanto,

Teorema 1.3.6 Se $f:[a, b] \rightarrow X$ e $a:[a, b] \rightarrow L(X, Y)$ são limitadas e se $D_{f}$ e $D_{\alpha}$ não têm pontos comuns, entào

(i) $f \in I^{\alpha}([a, b], X)$ implica que $f \in R^{\alpha}([a, b], X)$ e as integrais coincidem;

(ii) $a \in I^{f}([a . b] . X)$ implica que $a \in R^{f}([a, b], L(X, Y))$ e as integrais coincidem.

Prova (veja [H]. teor. I.1.2)

Para as integrais interiores e de Riemann-Stieltjes valem as seguintes propriedades:

(1) bilinearidacle, isto é. para $a, \beta:[a, b] \rightarrow L(X, Y)$ e $c \in \mathbb{R}$ temos:

se $f \in R^{\alpha}([a, b] . \mathcal{X}) \cap R^{\beta}([a, b] . X)$. entào $f \in R^{\alpha+\beta}([a, b], X)$ e

$$
\int_{a}^{b} d(\alpha+\beta)(t) f(t)=\int_{a}^{b} d \alpha(t) f(t)+\int_{a}^{b} d \beta(t) f(t),
$$

se $f \in R^{\beta}([a, b], X)$. entào $f \in R^{c \beta}([a, b] . X)$ e

$$
\int_{a}^{b} d(c \beta)(t) f(t)=c \int_{a}^{b} d \beta(t) f(t)
$$


se $f, g \in R^{\alpha}([a, b], X)$, entào $(f+g) \in R^{\alpha}([a, b], X)$ e

$$
\int_{a}^{b} d \alpha(t)(f+g)(t)=\int_{a}^{b} d \alpha(t) f(t)+\int_{a}^{b} d \alpha(t) g(t)
$$

se $f \in R^{\alpha}([a, b], X)$, então $c f \in R^{\alpha}([a, b], X)$ e

$$
\int_{a}^{b} d \alpha(t)(c f)(t)=c \int_{a}^{b} d \alpha(t) f(t)
$$

e o mesmo a.contece para as outras integrais;

(2) integrabilidade num intervalo implica integrabilidade nos subintervalos.

\section{Lema 1.3.7 (LEMA DE SACKS-HENSTOCK - para integrais de Riemann-Stieltjes)}

(i) Seja $f \in R^{\alpha}([a, b], X)$. Se para $\varepsilon>0, \delta>0$ é tal que $\forall D \in T D_{[a, b]} \operatorname{com} \Delta D<\delta$ temos

$$
\left\|s^{\alpha}(f, D)-\int_{a}^{b} d \alpha(t) f(t)\right\|<\varepsilon,
$$

então, para $a \leq c_{1} \leq \eta_{1} \leq d_{1} \leq c_{2} \leq \eta_{2} \leq d_{2} \leq \ldots \leq c_{k} \leq \eta_{k} \leq d_{k} \leq b$ com $\eta_{j} \in\left[c_{j}, d_{j}\right]$ e $d_{j}-c_{j}<\delta$ para cada $j$, temos que

$$
\left\|\sum_{j=1}^{k}\left\{\left[\alpha\left(d_{j}\right)-\alpha\left(c_{j}\right)\right] f\left(\eta_{j}\right)-\int_{c_{j}}^{d_{j}} d \alpha(t) f(t)\right\}\right\|<\varepsilon ;
$$

(ii) Seja $\alpha \in R^{f}([a, b], L(X, Y))$. Se para $\_>0$, o gauge $\delta$ de $[a, b]$ é tal que $\forall D \in T D_{[a, b]}$ $\delta$-fina temos

$$
\left\|s^{f}(\alpha, D)-\int_{a}^{b} \alpha(t) d f(t)\right\|<\varepsilon,
$$

então, para $a \leq c_{1} \leq \eta_{1} \leq d_{1} \leq c_{2} \leq \eta_{2} \leq d_{2} \leq \ldots \leq c_{k} \leq \eta_{k} \leq d_{k} \leq b \operatorname{com} \eta_{j} \in\left[c_{j}, d_{j}\right]$ e $d_{j}-c_{j}<\delta$ para cada $j$, temos que

$$
\left\|\sum_{j=1}^{k}\left\{\alpha\left(\eta_{j}\right)\left[f\left(d_{j}\right)-f\left(c_{j}\right)\right]-\int_{c_{j}}^{d_{j}} \alpha(t) d f(t)\right\}\right\|<\varepsilon .
$$

Prova(ídem à demonstração do Lema 2.1.6, pág 2S, fazendo $\delta$ constante.)

Assim como o Teorema 1.3.6, os dois próximos teoremas propõem condições para a existência das integrais de Riemann-Stieltjes. 
Teorema 1.3.8 Sejam $\alpha \in S V([a, b], L(X, Y))$ e $f \in \mathcal{C}([a, b], X)$. Então $f \in R^{\alpha}([a, b], X)$ com

$$
\left\|\int_{a}^{b} d \alpha(t) f(t)\right\| \leq S V(\alpha) \cdot\|f\|
$$

e, para $D \in T D_{[a, b]}$, temos que

$$
\left\|s^{\alpha}(f, D)-\int_{a}^{b} d \alpha(t) f(t)\right\| \leq S V(\alpha) \cdot \omega_{\Delta D}(f)
$$

Prova (veja [2], pág. 66, teor. 1.1).

De modo análogo, podemos mostrar o seguinte teorema:

Teorema 1.3.9 Sejam $\alpha \in \mathcal{C}([a, b], L(X, Y)) \in f \in B W([a, b], X)$. Então $\alpha \in R^{f}([a, b], L(X, Y))$ com

$$
\left\|\int_{a}^{b} \alpha(t) d f(t)\right\| \leq\|\alpha\| \cdot B W(f)
$$

e, para $D \in T D_{[a, b]}$, temos que

$$
\left\|s^{f}(\alpha, D)-\int_{a}^{b} \alpha(t) d f(t)\right\| \leq \omega_{\Delta D}(\alpha) \cdot B W(f) .
$$

Mais tarde, no parágrafo seguinte sobre integração por partes, demonstramos um teorema que diz que $\alpha \in R^{f}([a, b] . L(X, Y))$ se e somente se $f \in R^{\alpha}([a, b], X)$ (Teorema 1.4.1).

Definimos, agora, as funçòes integrais de $f \in R^{\alpha}([a, b], X)$ e de $\alpha \in R^{f}([a, b], L(X, Y))$ e damos propriedades para aquelas funçòes nos resultados que seguem.

\section{Definição 1.3.10 Temos:}

(i) se $f \in R^{\alpha}([a, b], X)$, entâo definimos a função $\tilde{f}_{\alpha}:[a, b] \rightarrow Y$ por

$$
\dot{f}_{a}(\xi)=\int_{a}^{\xi} d \alpha(t) f(t)
$$

$\left(\right.$ se $\alpha(t)=t$, entîo $\left.\tilde{f}_{\alpha}=\hat{f}\right)$ 
(ii) se $\alpha \in R^{f}([a, b], L(X, Y))$, entâo definimos a função $\tilde{\alpha}_{f}:[a, b] \rightarrow Y$ por

$$
\tilde{\alpha}_{f}(\xi)=\int_{a}^{\xi} \alpha(t) d f(t) .
$$

Teorema 1.3.11 Se $\alpha \in G^{\sigma}([a, b], L(X, Y))$ e $f \in R^{\alpha}([a, b], X)$, entẫo $\tilde{f}_{\alpha} \in G([a, b], Y)$ com $\tilde{f}_{\alpha}(\xi+)-\tilde{f}_{\alpha}(\xi)=[\alpha(\xi \dot{+})-\alpha(\xi)] f(\xi), \forall \xi \in[a, b[$

e

$$
\left.\left.\tilde{f}_{\alpha}(\xi)-\hat{f}_{\alpha}(\xi-)=[\alpha(\xi)-\alpha(\xi \dot{-})] f(\xi), \forall \xi \in\right] a, b\right]
$$

\section{Prova}

Por hipótese $f \in R^{\alpha}([a, b], X)$.

Portanto, $\forall \Xi>0, \exists \delta>0$ tal que $\forall D=\left(\xi_{i}, t_{i}\right) \in T D_{[a, b]}$ com $\Delta D<\delta$ temos que

$$
\left\|\sum_{i=1}^{|D|}\left[\alpha\left(t_{i}\right)-\alpha\left(t_{i-1}\right)\right] f\left(\xi_{i}\right)-\int_{a}^{b} d \alpha(t) f(t)\right\|<\frac{\varepsilon}{2} .
$$

Como $\alpha \in G^{\sigma}([a, b], L(X, Y))$, entào $\exists \alpha(\xi \dot{+}) \cdot x=(\alpha \cdot x)(\xi+), \forall x \in X, \forall \xi \in[a, b[$.

Entào, $\exists \varepsilon^{\prime}>0$ tal que para $0<h<\varepsilon^{\prime}$ temos que

$$
\|[\alpha(\xi+h)-\alpha(\xi \dot{+})] f(\xi)\|<\frac{\varepsilon}{2} .
$$

Tomando $\delta<\varepsilon^{\prime}$ e $h<\delta$ vem:

$$
\begin{gathered}
\left\|\dot{f}_{\alpha}(\xi+h)-\dot{f}_{\alpha}(\xi)-[\alpha(\xi \dot{+})-\alpha(\xi)] f(\xi)\right\|= \\
=\left\|\int_{\xi}^{\xi+h} d \alpha(t) f(t)-[\alpha(\xi \dot{+})-\alpha(\xi)] f(\xi)\right\| \leq \\
\leq\left\|\int_{\xi}^{\xi+h} d \alpha(t) f(t)-[\alpha(\xi+h)-\alpha(\xi)] f(\xi)\right\|+\|[\alpha(\xi+h)-\alpha(\xi)] f(\xi)-[\alpha(\xi \dot{+})-\alpha(\xi)] f(\xi)\|<
\end{gathered}
$$




$$
<\frac{\varepsilon}{2}+\|[\alpha(\xi+h)-\alpha(\xi \dot{+})] f(\xi)\|,
$$

pelo LEMA DE SACKS-HENSTOCK (Lema 1.3.7) e segue a tese.

A demonstraçào da outra igualdade é análoga.

Corolário 1 Se $\alpha \in \mathcal{C}^{\sigma}([a, b], L(X, Y))$ e $f \in R^{\alpha}([a, b], X)$, então $\tilde{f}_{\alpha} \in \mathcal{C}([a, b], Y)$.

Teorema 1.3.12 Se $f \in G([a, b], X)$ e $\alpha \in R^{f}([a, b], L(X, Y))$, então $\tilde{\alpha}_{f} \in G([a, b], Y)$ com

$$
\dot{\alpha}_{f}(\xi+)-\hat{\alpha}_{f}(\xi)=\alpha(\xi)[f(\xi+)-f(\xi)], \forall \xi \in[a, b[
$$

$e$

$$
\left.\left.\hat{\alpha}_{f}(\xi)-\grave{\alpha}_{f}(\xi-)=\alpha(\xi)[f(\xi)-f(\xi-)], \forall \xi \in\right] a, b\right] .
$$

\section{Prova}

Por hipótese $\alpha \in R^{f}([a, b], L(X, Y))$.

Portanto, $\forall \varepsilon>0, \exists \delta>0$ tal que $\forall D=\left(\xi_{i}, t_{i}\right) \in T D_{[a, b]} \operatorname{com} \Delta D<\delta$ temos que

$$
\left\|\sum_{i=1}^{|D|} \alpha\left(\xi_{i}\right)\left[f\left(t_{i}\right)-f\left(t_{i-1}\right)\right]-\int_{a}^{b} \alpha(t) d f(t)\right\|<\frac{\varepsilon}{2} .
$$

Como $f \in G([a, b], X)$. entào $\exists f(\xi+)=\lim _{h \backslash 0} f(\xi+h), \forall \xi \in[a, b[$.

Então, $\exists \varepsilon^{\prime}>0$ tal que para $0<h<\xi^{\prime}$ temos que

$$
\|f(\xi+h)-f(\xi+)\|<\frac{\varepsilon}{2\|\alpha(\xi)\|} .
$$

Tomando $\delta<\varepsilon^{\prime}$ e $h<\delta$ vem:

$$
\left\|\hat{\alpha}_{f}(\xi+h)-\dot{\alpha}_{f}(\xi)-\alpha(\xi)[f(\xi+)-f(\xi)]\right\|=
$$




$$
\begin{gathered}
=\left\|\int_{\xi}^{\xi+h} \alpha(t) d f(t)-\alpha(\xi)[f(\xi+)-f(\xi)]\right\| \leq \\
\leq\left\|\int_{\xi}^{\xi+h} \alpha(t) d f(t)-\alpha(\xi)[f(\xi+h)-f(\xi)]\right\|+\|\alpha(\xi)[f(\xi+h)-f(\xi+)]\|< \\
<\frac{\varepsilon}{2}+\|\alpha(\xi)\|\|f(\xi+h)-f(\xi+)\|,
\end{gathered}
$$

pelo LEMA DE SACKS-HENSTOCK (Lema 1.3.7) e segue a tese.

A demonstração da outra igualdade é análoga.

Corolário 1 Se $f \in \mathcal{C}([a, b], X)$ e $\alpha \in R^{f}([a, b], L(X, Y))$, então $\tilde{\alpha}_{f} \in \mathcal{C}([a, b], Y)$. 


\subsection{Integração por partes}

Dadas as funções $f:[a, b] \rightarrow X$ e $\alpha:[a, b] \rightarrow L(X, Y)$, vamos estudar condições necessárias para a validade da fórmula de integração por partes para as integrais de Riemann-Stieltjes. Também vamos explorar o caso em que ou $f$ ou $\alpha$ é uma função integral. Desta forma, obtemos as fórmulas de substituição para estas integrais.

Sejam, então, $f:[a, b] \rightarrow X$ e $\alpha:[a, b] \rightarrow L(X, Y)$. Chamamos fórmula de integração por partes à igualdade do teorema abaixo.

Teorema 1.4.1 $f \in R^{\alpha}([a, b], X)$ se $e$ só se $a \in R^{f}([a, b], L(X, Y))$ e temos que

$$
\int_{a}^{b} d \alpha(t) f(t)+\int_{a}^{b} \alpha(t) d f(t)=\alpha(b) f(b)-\alpha(a) f(a)
$$

Prova

$(\Longrightarrow)$ Por hipótese, $f \in R^{\alpha}([a, b], X)$.

Portanto, $\forall \varepsilon>0, \exists \delta>0$ tal que $\forall D=\left(\xi_{i}, t_{i}\right) \in T D_{[a, b]}$ com $\Delta D<\delta$ temos que

$$
\left\|\sum_{i=1}^{|D|}\left[\alpha\left(t_{i}\right)-\alpha\left(t_{i-1}\right)\right] f\left(\xi_{i}\right)-\int_{a}^{b} d \alpha(t) f(t)\right\|<\varepsilon .
$$

Daí,

$$
\begin{aligned}
& \left\|\left\{\alpha(b) f(b)-\alpha(a) f(a)-\int_{a}^{b} d \alpha(t) f(t)\right\}-\sum_{i=1}^{|D|} \alpha\left(\xi_{i}\right)\left[f\left(t_{i}\right)-f\left(t_{i-1}\right)\right]\right\|= \\
= & \left\|\sum_{i=1}^{|D|}\left[\alpha\left(t_{i}\right) f\left(t_{i}\right)-\alpha\left(t_{i-1}\right) f\left(t_{i-1}\right)\right]-\int_{a}^{b} d \alpha(t) f(t)-\sum_{i=1}^{|D|} \alpha\left(\xi_{i}\right)\left[f\left(t_{i}\right)-f\left(t_{i-1}\right)\right]\right\|= \\
= & \left\|\sum_{i=1}^{|D|}\left[\alpha\left(t_{i}\right)-\alpha\left(\xi_{i}\right)\right] . f\left(t_{i}\right)+\sum_{i=1}^{|D|}\left[\alpha\left(\xi_{i}\right)-\alpha\left(t_{i-1}\right)\right] f\left(t_{i-1}\right)-\int_{a}^{b} d \alpha(t) f(t)\right\|<\varepsilon,
\end{aligned}
$$


pois $\Delta D<\delta$ implica que $\left(t_{i}-t_{i-1}\right)<\delta, \forall i$ e, portanto, $\left(t_{i}-\xi_{i}\right)<\delta$ e $\left(\xi_{i}-t_{i-1}\right)<\delta, \forall i$.

$$
(\Longleftarrow) \text { Análoga. }
$$

Pelos Teoremas 1.3.6, 1.3.8 e 1.3.9 obtemos o seguinte corolário:

Corolário 1 Existem as integrais de Riemann-Stieltjes para as funções $f:[a, b] \rightarrow X$ e $\alpha:[a, b] \rightarrow L(X, Y)$ e vale a fórmula de integração por partes, se uma das condições abaixo são satisfeitas:

(i) $D_{\alpha}$ e $D_{f}$ nâo têm pontos comuns e $f \in I^{\alpha}([a, b], X)$ ou $\alpha \in I^{f}([a, b], L(X, Y))$;

(ii) $\alpha \in S V([a, b], L(X, Y))$ e $f \in \mathcal{C}([a, b], X)$;

(iii) $\alpha \in \mathcal{C}([a, b], L(X, Y)) \in f \in B W([a, b], X)$.

No caso geral, a fórmula de integraçào por partes não vale para as integrais interiores conforme mostra o exemplo seguinte:

Exemplo 3 Sejam $\alpha, f:[a, b] \rightarrow \mathbb{R}$ definidas por $\alpha=f=\chi_{\{a\}}$.

$\operatorname{Para} D^{\bullet}=\left(\xi_{i}, t_{i}\right) \in T D_{[a, b]}^{\bullet}$, temos:

$$
\left[\alpha\left(t_{i}\right)-\alpha\left(t_{i-1}\right)\right] . f\left(\xi_{i}\right)=0, \forall i \in \alpha\left(\xi_{i}\right)\left[f\left(t_{i}\right)-f\left(t_{i-1}\right)\right]=0, \forall i .
$$

Daí,

$$
\int_{a}^{b} \cdot d \alpha(t) f(t)=0 e \int_{a}^{b} \cdot \alpha(t) d f(t)=0
$$

E segue que

$$
0=\int_{a}^{b} \cdot d \alpha(t) f(t)+\int_{a}^{b} \cdot \alpha(t) d f(t) \neq \alpha(b) f(b)-\alpha(a) f(a)=1 .
$$

Agora consideremos o caso em que $\alpha=\hat{\beta}, \operatorname{com} \beta \in R([a, b], L(X, Y))$.

É fácil mostrarmos que 
Proposição 1.4.2 Para $\beta \in R([a, b], L(X, Y))$ e $\alpha=\tilde{\beta}$ temos que $\alpha \in A C([a, b], L(X, Y))$ e $V(\alpha) \leq(b-a)\|\beta\|$.

Então, pela Proposição 1.4.2 acima e pelo corolário do Teorema 1.4.1 é imediato o seguinte resultado para a fórmula de integração por partes:

Teorema 1.4.3 Sejam $\beta \in R([a, b], L(X, Y)), \alpha=\tilde{\beta}$ e $f \in \mathcal{C}([a, b], X)$. Então temos que $f \in R^{\alpha}([a, b], X), \alpha \in R^{f}([a, b], L(X, Y)) e$

$$
\int_{a}^{b} d \alpha(t) f(t)+\int_{a}^{b} \alpha(t) d f(t)=\alpha(b) f(b)-\alpha(a) f(a) .
$$

De modo mais geral, podemos tomar a : $[a, b] \rightarrow L(X, Y)$ uma função integral do tipo Riemann-Stieltjes, ou seja, $\alpha=\dot{\beta}_{\gamma} \operatorname{com} \gamma:[a, b] \rightarrow L(W, Y)$ e $\beta \in R^{\gamma}([a, b], L(X, W))$. Com isto, vamos obter um teorema para a fórmula de substituição.

Teorema 1.4.4 Sejam $\alpha:[a, b] \rightarrow L(W, Y), \beta \in R^{\alpha}([a, b], L(X, W)), \gamma=\tilde{\beta}_{\alpha}$ e seja também $f \in B V([a, b], X)$. Entâo $f \in R^{\gamma}([a, b], X)$ se e só se $\beta \cdot f \in R^{\alpha}([a, b], W)$. Neste caso,

$$
\int_{a}^{b} d \alpha(t) \beta(t) f(t)=\int_{a}^{b} d \gamma(t) f(t)
$$

\section{Prova}

Por hipótese, $\beta \in R^{\alpha}([a, b], L(X, W))$.

Portanto, $\forall \Xi>0, \exists \delta>0$ tal que $\forall D=\left(\xi_{i}, t_{i}\right) \in T D_{[a, b]} \operatorname{com} \Delta D<\delta$ temos que

$$
\left\|\sum_{i=j}^{|D|}\left\{\left[\alpha\left(t_{i}\right)-\alpha\left(t_{i-1}\right)\right] \beta\left(\xi_{i}\right)-\int_{t_{i-1}}^{t_{i}} d \alpha(t) \beta(t)\right\}\right\|<\varepsilon, \forall j \in\{1,2, \ldots,|D|\},
$$

pelo LEMA DE SACKS-HENSTOCK (Lema 1.3.7). 
Tomando as somas aproximadas de $\int_{a}^{b} d \alpha(t) \beta(t) f(t)$ e de $\int_{a}^{b} d \gamma(t) f(t)$ vem:

$$
\begin{aligned}
& \left\|\sum_{i=1}^{|D|}\left[\alpha\left(t_{i}\right)-\alpha\left(t_{i-1}\right)\right] \beta\left(\xi_{i}\right) f\left(\xi_{i}\right)-\sum_{i=1}^{|D|}\left[\gamma\left(t_{i}\right)-\gamma\left(t_{i-1}\right)\right] f\left(\xi_{i}\right)\right\|= \\
& =\left\|\sum_{i=1}^{|D|}\left\{\left[\alpha\left(t_{i}\right)-\alpha\left(t_{i-1}\right)\right] \beta\left(\xi_{i}\right)-\int_{t_{i-1}}^{t_{i}} d \alpha(t) \beta(t)\right\} f\left(\xi_{i}\right)\right\|=I .
\end{aligned}
$$

Para $\gamma_{i} \in L(X, Y)$ e $x_{i} \in X$ temos:

$$
\sum_{i=1}^{n} \gamma_{i} x_{i}=\left(\sum_{i=1}^{n} \gamma_{i}\right) x_{0}+\sum_{j=1}^{n}\left(\sum_{i=j}^{n} \gamma_{i}\right)\left(x_{j}-x_{j-1}\right)
$$

Então, fazendo $\gamma_{i}=\left[\alpha\left(t_{i}\right)-\alpha\left(t_{i-1}\right)\right] \beta\left(\xi_{i}\right)-\int_{t_{1-1}}^{t_{i}} d \alpha(t) \beta(t), x_{i}=f\left(\xi_{i}\right)$ e $x_{0}=f(a)$ temos:

$$
\begin{gathered}
I \leq\left\|\sum_{i=1}^{|D|}\left\{\left[\alpha\left(t_{i}\right)-\alpha\left(t_{i-1}\right)\right] \beta\left(\xi_{i}\right)-\int_{t_{i-1}}^{t_{i}} d \alpha(t) \beta(t)\right\}\right\|\|f(a)\|+ \\
+\sum_{j=1}^{|D|}\left\|\sum_{i=j}^{|D|}\left\{\left[\alpha\left(t_{i}\right)-\alpha\left(t_{i-1}\right)\right] \beta\left(\xi_{i}\right)-\int_{t_{i-1}}^{t_{i}} d \alpha(t) \beta(t)\right\}\right\|\left\|f\left(\xi_{j}\right)-f\left(\xi_{j-1}\right)\right\|< \\
<\varepsilon \cdot\|f(a)\|+\varepsilon \cdot V(f) .
\end{gathered}
$$

E segue a tese.

Agora, tomando $f$ como sendo uma funçào integral, chegamos ao teorema para a fórmula de substituiçào que segue:

Teorema 1.4.5 Sejam $a \in S V([a, b] . L(X, Y)), g:[a, b] \rightarrow W, f \in R^{g}([a, b], L(W, X))$, e $\beta=\tilde{f}_{g}:[a, b] \rightarrow X$. Entâo $\alpha \in R^{\beta}\left([a, b], L(X, Y)\right.$ se e só se $\alpha \cdot f \in R^{g}([a, b], Y)$. Neste caso,

$$
\int_{a}^{b} \alpha(t) f(t) d g(t)=\int_{a}^{b} \alpha(t) d \beta(t) .
$$




\section{Prova}

Por hipótese, $f \in R^{g}([a, b], L(W, X))$

Portanto, $\forall \varepsilon>0, \exists \delta>0$ tal que $\forall D=\left(\xi_{i}, t_{i}\right) \in T D_{[a, b]}$ com $\Delta D<\delta$ temos que

$$
\left\|\sum_{i=j}^{|D|}\left\{f\left(\xi_{i}\right)\left[g\left(t_{i}\right)-g\left(t_{i-1}\right)\right]-\int_{t_{-1}}^{t_{i}} f(t) d g(t)\right\}\right\|<\varepsilon, \forall j \in\{1,2, \ldots,|D|\},
$$

pelo LEMA DE SACKS-HENSTOCK (Lema 1.3.7).

Tomando as somas aproximadas de $\int_{a}^{b} \alpha(t) f(t) d g(t)$ e de $\int_{a}^{b} \alpha(t) d \beta(t)$ vem:

$$
\begin{aligned}
& \left\|\sum_{i=1}^{|D|} \alpha\left(\xi_{i}\right) f\left(\xi_{i}\right)\left[g\left(t_{i}\right)-g\left(t_{i-1}\right)\right]-\sum_{i=1}^{|D|} \alpha\left(\xi_{i}\right)\left[\beta\left(t_{i}\right)-\beta\left(t_{i-1}\right)\right]\right\|= \\
& =\left\|\sum_{i=1}^{|D|} \alpha\left(\xi_{i}\right)\left\{f\left(\xi_{i}\right)\left[g\left(t_{i}\right)-g\left(t_{i-1}\right)\right]-\int_{t_{i-1}}^{t_{i}} f(t) d g(t)\right\}\right\|=I .
\end{aligned}
$$

Para $\alpha_{i} \in L(X, Y)$ e $x_{i} \in X$ temos que

$$
\sum_{i=1}^{n} \alpha_{i} x_{i}=\alpha_{0}\left(\sum_{i=1}^{n} x_{i}\right)+\sum_{j=1}^{n}\left(\alpha_{j}-\alpha_{j-1}\right)\left(\sum_{i=j}^{n} x_{i}\right) .
$$

Entào, fazendo $x_{i}=f\left(\xi_{i}\right)\left[g\left(t_{i}\right)-g\left(t_{i-1}\right)\right]-\int_{t_{-}-1}^{t_{2}} f(t) d g(t), \alpha_{i}=\alpha\left(\xi_{i}\right)$ e $\alpha_{0}=\alpha(a)$ temos:

$$
\left\|\sum_{i=j}^{|D|}\left\{f\left(\xi_{i}\right)\left[g\left(t_{i}\right)-g\left(t_{i-1}\right)\right]-\int_{t_{i-1}}^{t_{i}} f(t) d g(t)\right\}\right\|=\left\|\sum_{i=j}^{|D|} x_{i}\right\|<\varepsilon, \forall j,
$$

pela integrabilidade de $f$.

Logo,

$$
\begin{gathered}
I \leq\|\alpha(a)\|\left\|\sum_{i=1}^{|D|} x_{i}\right\|+\left\|\sum_{j=1}^{|D|}\left[\alpha\left(\xi_{j}\right)-\alpha\left(\xi_{j-1}\right)\right]\left(\sum_{i=j}^{|D|} x_{i}\right)\right\|< \\
<\|\alpha(a)\| \cdot \varepsilon+S V(\alpha) \cdot \varepsilon .
\end{gathered}
$$

E segue a tese. 


\section{Capítulo 2}

\section{As Integrais Vetoriais de Gauge}

Nos parágrafos 2.1 e 2.2 deste capítulo, vamos definir e dar algumas propriedades das integrais vetoriais de Kurzweil e de Henstock, respectivameste. Uma destas propriedades é enunciada no LEMA DE SACKS-HENSTOCK para integrais de Kurzweil e de Henstock que é importante para a generalizaçào dos resultados obtidos no capítulo anterior para as integrais de RiemannStielt.jes, incluindo as fórmulas de substituição.

No parágrafo 2.3, vamos ver uma propriedade particularmente interessante de ambos os espaços das funções Kurzweil integráveis e das funções Henstock integráveis: tais espaços contém suas integrais impróprias. 


\subsection{A Integral Vetorial de Kurzweil}

Definição 2.1.1 Vamos definir:

(i) chamamos gauge de $[a, b]$ uma função $\delta:[a, b] \rightarrow \mathbb{R}_{+}^{*}$;

(ii) dada $D=\left(\xi_{i}, t_{i}\right) \in T D_{[a, b]}$, dizemos que $D$ é $\delta$-fina se e só se $\left[t_{i-1}, t_{i}\right] \subset B_{\delta\left(\xi_{i}\right)}\left(\xi_{i}\right)$ para cada $i$;

(iii) dados $c \in[a, b]$ e $\delta$ um gauge de $[a, b]$, dizemos que $\delta$ é c-especial ou $\delta$ é especial relativamente à $\mathrm{c}$, se $c \in B_{\delta(\xi)}(\xi)$ implica que $\xi=c$.

Exemplo 1 Seja $c \in[a, b]$. Entào qualquer gauge $\delta$ de $[a, b]$ tal que $\delta(\xi) \leq|\xi-c|, \forall \xi \neq c e ́$ c-especial.

Daí, podemos generalizar este exemplo como segue:

Proposição 2.1.2 Dado um número finito de pontos de $[a, b]$, existe um gauge de $[a, b]$ que é especial relativamente à estes pontos.

Teorema 2.1.3 Sâo equivalentes as afirmaçôes:

(i) para todo gauge $\delta$ de $[a, b]$, existe $D \in T D_{[a, b]} \delta$-fina;

(ii) toda cobertura aberta de $[a, b]$ admite uma subcobertura finita. 


\section{Prova}

(i) $\Longrightarrow($ ii $)$

Seja $B=\left\{I_{j} ; j \in A\right\}$ uma cobertura aberta de $[a, b]$.

Podemos admitir, sem perda de generalidade, que os $I_{j}$ 's são intervalos abertos não vazios.

Definamos um gauge $\delta$ de $[a, b]$ da seguinte maneira: para cada $\xi \in[a, b]$, fixemos um $I_{j}$ tal que $\xi \in I_{j}$ e tomemos $\delta(\xi)$ tal que $B_{\delta(\xi)}(\xi) \subset I_{j}$.

Por hipótese, $\exists D=\left(\xi_{i}, t_{i}\right) \in T D_{[a, b]} \delta$-fina.

Para cada $i \in\{1,2, \ldots,|D|\}$, tomemos $j_{i} \in A$ tal que $I_{j_{i}}$ é o intervalo fixado à pouco para $\xi_{i}$.

Fazendo $A^{\prime}=\left\{j_{i} \in A ; i \in\{1,2, \ldots,|D|\}\right\}$ temos que $B^{\prime}=\left\{I_{j} ; j \in A^{\prime}\right\}$ é uma subcobertura finita de $B$ para $[a, b]$.

(ii) $\Longrightarrow$ (i)

Seja $\delta$ um gauge de $[a, b]$.

Portanto, $B=\left\{B_{\delta(\xi)}(\xi) ; \xi \in[a, b]\right\}$ é uma cobertura aberta de $[a, b]$, que admite uma subcobertura finita $B=\left\{B_{\delta\left(\xi^{\jmath}\right)}\left(\xi^{j}\right) ; j=1,2, \ldots, m\right\}$, por hipótese.

Tomemos $t_{0}=a$.

Indicamos por $\xi_{1}$ um ponto $\xi^{i}$ tal que $\xi^{i}+\delta\left(\xi^{i}\right)$ seja máximo entre os $\xi^{j}$ 's tais que $a \in$ $B_{\delta\left(\xi^{\jmath}\right)}\left(\xi^{j}\right)$.

Indiquemos por $\xi_{2}$ um ponto $\xi^{i}$ tal que $\xi^{i}+\delta\left(\xi^{i}\right)$ seja máximo entre os $\xi^{j}$ 's tais que $\xi_{1}+\delta\left(\xi_{1}\right) \in$ $B_{\delta\left(\xi^{\jmath}\right)}\left(\xi^{j}\right)$.

Tomando $t_{1} \in B_{\delta\left(\xi_{1}\right)}\left(\xi_{1}\right) \cap B_{\delta\left(\xi_{2}\right)}\left(\xi_{2}\right)$ tal que $\xi_{1} \leq t_{1} \leq \xi_{2}$ temos: $\xi_{1} \in\left[t_{0}, t_{1}\right] \subset B_{\delta\left(\xi_{1}\right)}\left(\xi_{1}\right)$. 
Indicamos por $\xi_{3}$ um ponto $\xi^{i}$ tal que $\xi^{i}+\delta\left(\xi^{i}\right)$ seja máximo entre os $\xi^{j}$ 's tais que $\xi_{2}+\delta\left(\xi_{2}\right) \in$ $B_{\delta\left(\xi^{\jmath}\right)}\left(\xi^{j}\right)$.

Tomando $t_{2} \in B_{\mathcal{S}\left(\xi_{2}\right)}\left(\xi_{2}\right) \cap B_{\delta\left(\xi_{3}\right)}\left(\xi_{3}\right)$ tal que $\xi_{2} \leq t_{1} \leq \xi_{3}$ temos: $\xi_{2} \in\left[t_{1}, t_{2}\right] \subset B_{\delta\left(\xi_{2}\right)}\left(\xi_{2}\right)$.

Repetindo este processo obtemos um número finito de $\xi_{n}$ 's.

Quando $\xi_{n}+\delta\left(\xi_{n}\right)>b$ o processo terminou e definimos $t_{n}=b$.

Então, $D=\left(\xi_{i}, t_{i}\right) \in T D_{[a, b]}$ e é $\delta$-fina.

Daí, segue o

Teorema 2.1.4 (LEMA DE COUSIN) Para todo gauge $\delta$ de $[a, b]$, existe $D \in T D_{[a, b]} \delta$-fina.

Então, o Teorema 2.1.3 equivale à dizer que o Lema de Cousin caracteriza o corpo dos números reais (veja [5]. apêndice C).

Vamos sempre admitir $a:[a, b] \rightarrow L(X, Y)$ e $f:[a, b] \rightarrow X$ se não houver menção explícita em contrário.

\section{Definição 2.1.5 Definimos:}

(i) $f$ é Kurzweil integrável em relação à $\alpha$, e denotamos $f \in K^{\alpha}([a, b], X)$, se existe $I \in Y$ satisfazendo:

$\forall \varepsilon>0, \exists \delta$ gauge de $[a, b]$ tal que $\forall D \in T D_{[a, b]} \delta$-fina temos que $\left\|s^{\alpha}(f, D)-I\right\|<\varepsilon$.

Neste caso, escrevemos $I={ }^{k \cdot b} d \alpha(t) f(t)$ (quando $\alpha(t)=t$, escrevemos $K([a, b], X) e$ $\left.K_{a}^{b} d a(t) f(t)=\int_{a}^{k} f(t) d t\right)$. 
(ii) $\alpha$ é Kurzweil integrável em relação à $f$, e denotamos $\alpha \in K^{f}([a, b], L(X, Y))$, se existe $I \in Y$ satisfazendo:

$\forall \varepsilon>0, \exists \delta$ gauge de $[a, b]$ tal que $\forall D \in T D_{[a, b]} \delta$-fina temos que $\left\|s^{f}(\alpha, D)-I\right\|<\varepsilon$. Neste caso, escrevemos $I={ }^{k} \int_{a}^{b} \alpha(t) d f(t)$.

Em ambos os espaços $K^{\alpha}([a, b], X)$ e $K^{f}([a, b], L(X, Y))$ valem as seguintes propriedades: (1) bilinearidade da integral;

(2) integrabilidade num intervalo implica integrabilidade nos subintervalos e vice-versa, isto é, se $f \in K^{\alpha}([a, b], X)$, entào $f \in K^{\alpha}([u, v], X), \forall[u, v] \subset[a, b]$ e, se $f \in K^{\alpha}([a, c], X)$ e $f \in K^{\alpha}([c, b], X)$, entào $f \in K^{\alpha}([a, b], X)$ com

$$
\int_{a}^{k b^{b}} d \alpha(t) f(t)=\int_{a}^{k j} d \alpha(t) f(t)+\int_{c}^{K k^{b}} d \alpha(t) f(t)
$$

e o mesmo vale para a outra integral.

Seguimos com o LEMA DE SACKS-HENSTOCK, ferramenta muito útil para a demonstração da majoria dos resultados deste e do próximo parágrafos.

\section{Lema 2.1.6 (LEMA DE SACKS-HENSTOCK)}

(i) Seja $f \in K^{\circ}([a, b], X)$. Se para $=>0$, o gauge $\delta$ de $[a, b]$ é tal que $\forall D \in T D_{[a, b]}$ $\delta$-fina temos

$$
\left\|s^{\alpha}(f, D)-\int_{a}^{k b} d \alpha(t) f(t)\right\|<\varepsilon,
$$

entiò, para $a \leq c_{1} \leq \eta_{1} \leq d_{1} \leq c_{2} \leq \eta_{2} \leq d_{2} \leq \ldots \leq c_{k} \leq \eta_{k} \leq d_{k} \leq b$ com $\eta_{j} \in\left[c_{j}, d_{j}\right] \subset B_{\delta\left(\eta_{j}\right)}\left(\eta_{j}\right)$ para cada $j$, temos que

$$
\left\|\sum_{j=1}^{k}\left\{\left[\alpha\left(d_{j}\right)-\alpha\left(c_{j}\right)\right] f\left(\eta_{j}\right)-\int_{c_{j}}^{k_{j}} d \alpha(t) f(t)\right\}\right\|<\varepsilon ;
$$


(ii) Seja $\alpha \in K^{f}([a, b], L(X, Y))$. Se para $\varepsilon>0$, o gauge $\delta$ de $[a, b]$ é tal que $\forall D \in T D_{[a, b]}$ $\delta$-fina temos

$$
\left\|s^{f}(\alpha, D)-\int_{a}^{K p} \alpha(t) d f(t)\right\|<\varepsilon
$$

entâo, para $a \leq c_{1} \leq \eta_{1} \leq d_{1} \leq c_{2} \leq \eta_{2} \leq d_{2} \leq \ldots \leq c_{k} \leq \eta_{k} \leq d_{k} \leq b$ com $\eta_{j} \in\left[c_{j}, d_{j}\right] \subset B_{\delta\left(\eta_{j}\right)}\left(\eta_{j}\right)$ para cada $j$, temos que

$$
\left\|\sum_{j=1}^{k}\left\{\alpha\left(\eta_{j}\right)\left[f\left(d_{j}\right)-f\left(c_{j}\right)\right]-\int_{c_{j}}^{k_{j}} \alpha(t) d f(t)\right\}\right\|<\varepsilon .
$$

\section{Prova}

Esta demonstraçào é baseada em [\$], pág. 90 .

Vamos fazer a demonstraçào de (i). A demonstração de (ii) é análoga.

Para cada $j$, seja $J_{j}=\left[c_{j}, d_{j}\right]$.

O conjunto $[a, b] \backslash \cup_{j=1}^{k} J_{j}$ consiste de um número finito de intervalos de $[a, b]$.

Sejam $K_{1}, K_{2}, \ldots, K_{m}$ os fechos de cada um destes intervalos.

Como $f \in K^{\alpha}([a, b], X)$, entào $f \in K^{\alpha}\left(K_{p}, X\right), \forall p=1,2, \ldots, m$.

Portanto, dado $\varepsilon^{\prime}>0, \exists \delta^{\prime}$ gauge de $K_{p}, \operatorname{com} \delta^{\prime}(\xi) \leq \delta(\xi)$ para cada $\xi \in K_{p}$, tal que $\forall D_{p}=\left(\xi_{p_{i}}, t_{p_{1}}\right) \in T D_{K_{p}}, \delta^{\prime}$-fina temos que

$$
\left\|\sum_{i=1}^{\left|D_{p}\right|}\left[\alpha\left(t_{p_{t}}\right)-\alpha\left(t_{p_{1-1}}\right)\right] f\left(\xi_{p_{t}}\right)-\int_{K_{p}}^{K} d \alpha(t) f(t)\right\|<\frac{\varepsilon^{\prime}}{m}, \forall p .
$$

Para cada $j, D_{j}^{\prime}=\left\{\left(\eta_{j}, d_{j}\right)\right\} \in T D_{J}$, e é $\delta$-fina.

Daí, $D=\left(\cup_{j=1}^{k} D_{j}^{\prime}\right) \cup\left(\cup_{p=1}^{m} D_{p}\right) \in T D_{[a, b]}$ e é $\delta$-fina.

Então. 


$$
\left\|s^{\alpha}(f, D)-\int_{a}^{k^{b}} d \alpha(t) f(t)\right\|<\varepsilon
$$

$\mathrm{e}$

$$
\begin{gathered}
\left\|s^{\alpha}(f, D)-\int_{a}^{K b} d \alpha(t) f(t)\right\| \geq \\
\geq\left\|\sum_{j=1}^{k}\left\{\left[\alpha\left(d_{j}\right)-\alpha\left(c_{j}\right)\right] f\left(\eta_{j}\right)-\int_{J_{j}}^{K} d \alpha(t) f(t)\right\}\right\|- \\
-\left\|\sum_{p=1}^{m}\left\{\sum_{i=1}^{\left|D_{p}\right|}\left[\alpha\left(t_{p_{i}}\right)-\alpha\left(t_{p_{i-1}}\right)\right] f\left(\xi_{p_{i}}\right)-\int_{K_{p}}^{K} d \alpha(t) f(t)\right\}\right\| .
\end{gathered}
$$

Logo,

$$
\begin{gathered}
\left\|\sum_{j=1}^{k}\left\{\left[\alpha\left(d_{j}\right)-\alpha\left(c_{j}\right)\right] f\left(\eta_{j}\right)-\int_{J_{j}}^{K} d \alpha(t) f(t)\right\}\right\|< \\
<\varepsilon+\sum_{p=1}^{m}\left\|\sum_{i=1}^{\left|D_{p}\right|}\left[\alpha\left(t_{p_{i}}\right)-\alpha\left(t_{p_{t-1}}\right)\right] f\left(\xi_{p_{i}}\right)-\int_{K_{p}}^{K j} d \alpha(t) f(t)\right\|< \\
<\varepsilon+m \cdot \frac{\varepsilon^{\prime}}{m}=\varepsilon+\varepsilon^{\prime}, \forall \varepsilon^{\prime}>0 .
\end{gathered}
$$

Assim,

$$
\left\|\sum_{j=1}^{k}\left\{\left[\alpha\left(d_{j}\right)-\alpha\left(c_{j}\right)\right] f\left(\eta_{j}\right)-\int_{c_{j}}^{k d_{j}} d \alpha(t) f(t)\right\}\right\| \leq \varepsilon .
$$

As funçòes Riemann-Stieltjes integráveis sào o caso particular das integrais vetoriais de Kurzweil onde o gauge é constante.

Portanto,

\section{Teorema 2.1.7 Temos:}

(i) $R^{\alpha}([a, b], X) \subset K^{\alpha}([a, b], X)$ e. se $f \in R^{\alpha}([a, b], X)$, entâa

$$
\int_{a}^{b} d \alpha(t) f(t)=\int_{a}^{k b} d \alpha(t) f(t)
$$


(ii) $R^{f}\left([a, b], L(X, Y) \subset K^{f}([a, b], L(X, Y))\right.$ e, se $\alpha \in R^{f}([a, b], L(X, Y)$, então

$$
\int_{a}^{b} \alpha(t) d f(t)=\int_{a}^{k} \alpha(t) d f(t)
$$

Prova (basta vermos que, para $\delta>0, \delta$ da definição das integrais de Riemann-Stieltjes, tomamos um gauge $\delta_{0}$ de $[a, b]$ constantemente igual à $\frac{\delta}{2}$, e segue que $\forall D \in T D_{[a, b]} \delta_{0}$-fina é tal que $\Delta D<\delta)$.

\section{Corolário 1 Temos:}

(i) se $f \in R^{\alpha}([a, b], X)$, então

$$
\int_{a}^{b} d \alpha(t) f(t)=\int_{a}^{b} \cdot d \alpha(t) f(t)=\int_{a}^{k b} d \alpha(t) f(t)
$$

(ii) se $\alpha \in R^{f}([a, b], L(X, Y)$, entâo

$$
\int_{a}^{b} \alpha(t) d f(t)=\int_{a}^{b} \cdot \alpha(t) d f(t)=\int_{a}^{K^{b}} \alpha(t) d f(t)
$$

Se entretanto não tivermos $f \in R^{\alpha}([a, b], X)$, por exemplo, então pode acontecer de termos $f \in K^{\alpha}([a, b], X) \cap I^{\alpha}([a, b] . X)$, mas $\int_{a}^{k} d \alpha(t) f(t) \neq \int_{a}^{b} \cdot d \alpha(t) f(t)$, conforme mostra o exemplo a seguir. E o mesmo pode ocorrer com a outra integral, se $\alpha \notin R^{f}([a, b], L(X, Y)$. Vejamos:

Exemplo 2 Sejam $f . a:[a, b] \rightarrow \mathbb{R}$ definidas por $f=\alpha=\chi_{] a, b]}$.

Seja $D=\left(\xi_{i}, t_{i}\right) \in T D_{[a, b]}$.

Entào, $\left[a\left(t_{i}\right)-\alpha\left(t_{i-1}\right)\right] . f\left(\xi_{i}\right)=\left\{\begin{array}{l}1 ; i=1 \in \xi_{1}>a \\ 0 ; i \neq 1 \text { ou } i=1 \text { e } \xi_{1}=a\end{array}\right.$.

Portanto.

$$
\nexists \int_{a}^{b} d \alpha(t) f(t), \text { mas } \exists \int_{a}^{b} \cdot d \alpha(t) f(t)=1 .
$$

Por outro lado. tomando um gauge $\delta$ de $[a, b]$ que seja a-especial temos:

$$
\forall D=\left(\xi_{i}, t_{i}\right) \in T D_{[a, b]} \delta \text {-fina é tal que } \xi_{1}=a .
$$


Logo,

$$
\exists \int_{a}^{K b} d \alpha(t) f(t)=0
$$

Pelo Teorema 2.1.7, não há confusão de notação se definirmos:

Definição 2.1.8 Temos:

(i) para $f \in K^{\alpha}([a, b], X)$, definimos a função $\tilde{f}_{\alpha}:[a, b] \rightarrow X$ por

$$
\hat{f}_{\alpha}(\xi)=\int_{a}^{K} d \alpha(t) f(t)
$$

$\left(\right.$ se $\alpha(t)=t$, escrevemos $\left.\tilde{f}_{o}=\dot{f}\right)$;

(ii) para $\alpha \in K^{f}([a, b], L(X, Y))$. definimos a função $\tilde{\alpha}_{f}:[a, b] \rightarrow L(X, Y)$ por

$$
\tilde{\alpha}_{f}(\xi)=\int_{a}^{k \xi} \alpha(t) d f(t)
$$

Os teoremas que seguem dão propriedades das funções $\tilde{f}_{\alpha}$ e $\tilde{\alpha}_{f}$.

Teorema 2.1.9 Se $f \in G([a, b], X)$ e $\alpha \in K^{f}([a, b], L(X, Y))$, entâo $\tilde{\alpha}_{f} \in G([a, b], Y)$ com

$$
\dot{\alpha}_{f}(\xi+)-\dot{\alpha}_{f}(\xi)=\alpha(\xi)[f(\xi+)-f(\xi)], \forall \xi \in[a, b[
$$

$e$

$$
\left.\left.\tilde{\alpha}_{f}(\xi)-\dot{\alpha}_{f}(\xi-)=\alpha(\xi)[f(\xi)-f(\xi-)], \forall \xi \in\right] a, b\right]
$$

\section{Prova}

Por hipótese, $\alpha \in K^{*}\left([a, b], L\left(X, Y^{*}\right)\right)$.

Portanto, $\forall \varepsilon>0, \exists \delta$ gauge de $[a, b]$ tal que $\forall D=\left(\xi_{i}, t_{i}\right) \in T D_{[a, b]} \delta$-fina temos que

$$
\left\|\sum_{i=1}^{|D|} \alpha\left(\xi_{i}\right)\left[f\left(t_{i}\right)-f\left(t_{i-1}\right)\right]-\int_{a}^{k b} \alpha(t) d f(t)\right\|<\frac{\varepsilon}{2} .
$$


Seja $\xi \in[a, b[$ arbitrário.

Como $f \in G([a, b], X)$ então existe $f(\xi+)$.

Logo, $\exists \varepsilon^{\prime}>0$ tal que para $0<h<\varepsilon^{\prime}$ temos que $\|f(\xi+h)-f(\xi+)\|<\min \left\{\frac{\varepsilon}{2}, \frac{\varepsilon}{2 \cdot\|\alpha(\xi)\|}\right\}$.

Sejam $\delta(\xi)<\varepsilon^{\prime}$ e $0<h<\delta(\xi)$.

Então, pelo LEMA DE SACKS-HENSTOCK (Lema 2.1.6), temos que

$$
\left\|\alpha(\xi)[f(\xi+h)-f(\xi)]-\int_{\xi}^{K \xi+h} \alpha(t) d f(t)\right\| \leq \frac{\varepsilon}{2} .
$$

Assim,

$$
\begin{gathered}
\left\|\tilde{\alpha}_{f}(\xi+h)-\dot{\alpha}_{f}(\xi)-\alpha(\xi)[f(\xi+)-f(\xi)]\right\|=\left\|\int_{\xi}^{K \xi+h} \alpha(t) d f(t)-\alpha(\xi)[f(\xi+)-f(\xi)]\right\| \leq \\
\leq\left\|\int_{\xi}^{K \xi+h} \alpha(t) d f(t)-\alpha(\xi)[f(\xi+h)-f(\xi)]\right\|+ \\
+\|\alpha(\xi)[f(\xi+h)-f(\xi)]-\alpha(\xi)[f(\xi+)-f(\xi)]\| \leq \\
\leq \frac{\varepsilon}{2}+\|\alpha(\xi)\|\|f(\xi+h)-f(\xi+)\|<\varepsilon .
\end{gathered}
$$

Portanto, existe $\hat{a_{f}}(\xi+)=\tilde{a}_{f}(\xi)+\alpha(\xi)[f(\xi+)-f(\xi)]$.

Analogamente, prova-se que existe $\hat{\alpha_{f}}(\xi-)$ satisfazendo a igualdade do enunciado.

Corolário 1 Se $f \in \mathcal{C}([a, b], X)$ e $a \in K^{f}([a, b], L(X, Y))$, então $\tilde{\alpha}_{f} \in \mathcal{C}([a, b], Y)$.

Teorema 2.1.10 Se $a \in G^{\sigma}([a, b], L(X, Y))$ ef $f \in K^{\alpha}([a, b], X)$, então $\hat{f}_{\alpha} \in G([a, b], Y)$ com

$$
\dot{f}_{\mathrm{a}}(\xi+)-\hat{f}_{\mathrm{a}}(\xi)=[\alpha(\xi \dot{+})-\alpha(\xi)] f(\xi), \forall \xi \in[a, b[
$$

$e$

$$
\left.\left.\dot{f}_{\alpha}(\xi)-\dot{f}_{\alpha}(\xi-)=[\alpha(\xi)-\alpha(\xi \dot{-})] f(\xi), \forall \xi \in\right] a, b\right]
$$




\section{Prova}

Por hipótese, $f \in K^{a}([a, b], X)$.

Portanto, $\forall \varepsilon>0, \exists \delta$ gauge de $[a, b]$ tal que $\forall D=\left(\xi_{i}, t_{i}\right) \in T D_{[a, b]} \delta$-fina temos que

$$
\left\|\sum_{i=1}^{|D|}\left[\alpha\left(t_{i}\right)-\alpha\left(t_{i-1}\right)\right] f\left(\xi_{i}\right)-\int_{a}^{b} d \alpha(t) f(t)\right\|<\frac{\varepsilon}{2} .
$$

Seja $\xi \in[a, b[$ arbitrário.

Como $\alpha \in G^{\sigma}([a, b], L(X, Y))$ então existe $(\alpha \cdot x)(\xi+), \forall x \in X$.

Em particular, $\exists \varepsilon^{\prime}>0$ tal que para $0<h<\varepsilon^{\prime}$ temos que $\|[\alpha(\xi+h)-\alpha(\xi \dot{+})] f(\xi)\|<\frac{\varepsilon}{2}$.

Sejam $\delta(\xi)<\varepsilon^{\prime}$ e $0<h<\delta(\xi)$

Entâo, pelo LEMA DE SACKS-HENSTOCK (Lema 2.1.6), temos que

$$
\left\|[\alpha(\xi+h)-\alpha(\xi)] f(\xi)-\int_{\xi}^{k+h} d \alpha(t) f(t)\right\| \leq \frac{\varepsilon}{2} .
$$

Assim,

$$
\begin{gathered}
\left\|\tilde{f}_{\alpha}(\xi+h)-\hat{f}_{\alpha}(\xi)-[\alpha(\xi \dot{+})-\alpha(\xi)] f(\xi)\right\|=\left\|\int_{\xi}^{K \xi+h} d \alpha(t) f(t)-[\alpha(\xi \dot{+})-\alpha(\xi)] f(\xi)\right\| \leq \\
\leq\left\|\int_{\xi}^{K \xi+h} d \alpha(t) f(t)-[\alpha(\xi+h)-\alpha(\xi)] f(\xi)\right\|+ \\
+\|[\alpha(\xi+h)-\alpha(\xi)] f(\xi)-[\alpha(\xi \dot{+})-\alpha(\xi)] f(\xi)\|<\frac{\varepsilon}{2}+\frac{\varepsilon}{2}=\varepsilon .
\end{gathered}
$$

Portanto, existe $\hat{f}_{\alpha}(\xi+)=\tilde{f}_{\alpha}(\xi)+[\alpha(\xi \dot{+})-\alpha(\xi)] f(\xi)$.

Analogamente, prova-se que existe $\hat{f}_{\alpha}(\xi-)$ satisfazendo a igualdade do enunciado.

Corolário 1 Se $a \in \mathcal{C}^{\sigma}([a, b], L(X, Y))$ e $f \in K^{\alpha}([a, b], X)$, então $\tilde{f}_{\alpha} \in \mathcal{C}([a, b], Y)$. 
Para $\hat{\alpha}_{f}, \operatorname{com} f=\tilde{h}_{g}$ e, para $\tilde{f}_{\alpha}, \operatorname{com} \alpha=\tilde{g}_{\beta}$ temos as seguintes propriedades que podem ser demonstrados através de procedimento análogo ao usado nas provas dos Teoremas $2.1 .9 \mathrm{e}$ 2.1.10:

Teorema 2.1.11 Sejam $g:[a, b] \rightarrow W, h \in K^{g}([a, b], L(W, X)), f=\tilde{h}_{g}:[a, b] \rightarrow X e$ $\alpha \in K^{f}([a, b], L(X, Y))$. Se $g \in G([a, b], W)$, então $\tilde{\alpha}_{f} \in G([a, b], Y)$ com

$$
\dot{\alpha}_{f}(\xi+)-\tilde{\alpha}_{f}(\xi)=\alpha(\xi) h(\xi)[g(\xi+)-g(\xi)], \forall \xi \in[a, b[
$$

e

$$
\left.\left.\tilde{\alpha}_{f}(\xi)-\tilde{\alpha}_{f}(\xi-)=\alpha(\xi) h(\xi)[g(\xi)-g(\xi-)], \forall \xi \in\right] a, b\right] .
$$

Corolário 1 Com as hipóteses do teorema, se $g \in \mathcal{C}([a, b], W)$, então $\tilde{\alpha}_{f} \in \mathcal{C}([a, b], Y)$.

Teorema 2.1.12 Sejam $\beta:[a, b] \rightarrow L(X, Y), g \in K^{\beta}([a, b], L(W, X)), \alpha=\tilde{g}_{\beta}:[a, b] \rightarrow$ $L(W, Y)$ e $f \in K^{\alpha}([a, b], W)$. Se $\beta \in G^{\sigma}([a, b], L(X, Y))$, então $\tilde{f}_{\alpha} \in G([a, b], Y)$ com

$$
\dot{f}_{\alpha}(\xi+)-\dot{f}_{\alpha}(\xi)=[\beta(\xi \dot{+})-\beta(\xi)] g(\xi) f(\xi), \forall \xi \in[a, b[
$$

$\epsilon$

$$
\left.\left.\dot{f}_{\alpha}(\xi)-\dot{f}_{\alpha}(\xi-)=[\beta(\xi)-\beta(\xi-)] g(\xi) f(\xi), \forall \xi \in\right] a, b\right] .
$$

Corolário 1 Com as hipóteses do teorema. se $\beta \in \mathcal{C}^{\sigma}([a, b], L(X, Y))$, então $\tilde{f}_{\alpha} \in \mathcal{C}([a, b], Y)$.

Agora, mencionemos um resultado sobre a fórmula de integração por partes para a integral de Kurzweil. 
Teorema 2.1.13 Sejam $\alpha \in S V([a, b], L(X, Y))$ e $f \in K([a, b], X)$. Então $\alpha \cdot f \in K([a, b], Y)$ e vale

$$
\int_{a}^{\kappa p} \alpha(t) f(t) d t=\int_{a}^{b} \alpha(t) d \tilde{f}(t)=\alpha(b) \tilde{f}(b)-\alpha(a) \tilde{f}(a)-\int_{a}^{b} d \alpha(t) \tilde{f}(t) .
$$

Prova (veja [6], 1.15).

A fórmula de substituição não é necessariamente verdadeira, mesmo quando as duas integrais estào definidas. Vejamos o exemplo a seguir:

Exemplo 3 Sejam $Y=\mathbb{R}$ e $X=l_{2}([a, b])$.

Seja $f:[a, b] \rightarrow X$ definida por $f(t)=\epsilon_{t}$, isto é, $e_{t}(s)=\left\{\begin{array}{l}1, s=t \\ 0, s \neq t\end{array}\right.$

Seja. também, $\alpha:[a, b] \rightarrow X^{\prime \prime}$ dada por $\alpha(t)=\tilde{e}_{t}$, onde $\tilde{e}_{t}(x)=<e_{t}, x>, \forall x \in X$.

Entào, $\alpha(t) f(t)=<\epsilon_{t}, \epsilon_{t}>=1 \epsilon$, portanto,

$$
\int_{a}^{l i b} \alpha(t) f(t) d t=\int_{a}^{b} d t=b-a .
$$

Por outro lado, dado $\varepsilon>0, \exists \delta>0$, com $\delta^{\frac{1}{2}}<\frac{\varepsilon}{(b-a)^{\frac{1}{2}}}$, tal que $\forall D=\left(\xi_{i}, t_{i}\right) \in T D_{[a, b]}$ com $\Delta D<\delta$ temos que

$$
\left\|\sum_{i=1}^{|D|} f\left(\xi_{i}\right)\left(t_{i}-t_{i-1}\right)-0\right\|_{2}=\left\|\sum_{i=1}^{|D|} \epsilon_{\xi_{i}}\left(t_{i}-t_{i-1}\right)\right\|_{2}=\left[\sum_{i=1}^{|D|}\left|t_{i}-t_{i-1}\right|^{2}\right]^{\frac{1}{2}},
$$

pela igualdade de Bessel.

Daí.

$$
\left[\sum_{i=1}^{|D|}\left|t_{i}-t_{i-1}\right|^{2}\right]^{\frac{1}{2}}=\left[\sum_{i=1}^{|D|}\left(t_{i}-t_{i-1}\right)\left(t_{i}-t_{i-1}\right)\right]^{\frac{1}{2}}<\delta^{\frac{1}{2}} \cdot \sum_{i=1}^{|D|}\left(t_{i}-t_{i-1}\right)^{\frac{1}{2}}=[\delta(b-a)]^{\frac{1}{2}}<\varepsilon .
$$

Evem que,

$$
0=\int_{a}^{b} f(t) d t=\int_{a}^{k b} f(t) d t
$$


Entâo, $\tilde{f}=0$ o que implica que $\int_{a}^{b} \alpha(t) d \tilde{f}(t)=0$.

Logo, para $b>$ a temos

$$
0<b-a=\int_{a}^{b} \alpha(t) f(t) d t \neq \int_{a}^{b} \alpha(t) d \tilde{f}(t)=0 .
$$

Agora, provemos que $\alpha \notin S V([a, b], L(X, Y))$.

Sejad: $a=t_{0}<t_{1}<\ldots<t_{n}=b$.

Então,

$$
\left\|\sum_{i=1}^{n}\left[\alpha\left(t_{i}\right)-\alpha\left(t_{i-1}\right)\right] \cdot e_{t_{i}}\right\|=\left\|\sum_{i=1}^{n}<e_{t_{i}}-e_{t_{i-1}}, e_{t_{i}}>\right\|=n .
$$

Logo, $S V(\alpha)=\infty$, porém $\|\alpha\| \leq 1$, ou seja, $\alpha$ é limitada mas não é de $S V([a, b], L(X, Y))$.

Mais geralmente temos o seguinte teorema para a fórmula de substituição:

Teorema 2.1.14 Sejam $\alpha \in S V([a, b], L(X, Y)), g:[a, b] \rightarrow W, f \in K^{g}([a, b], L(W, X))$, e $\beta:[a, b] \rightarrow X$ dada por $\beta=\tilde{f}_{g}$. Então $\alpha \in K^{\beta}([a, b], L(X, Y))$ se e somente se $\alpha \cdot f \in K^{g}([a, b], Y)$. Neste caso,

$$
\int_{a}^{k p} \alpha(t) f(t) d g(t)=\int_{a}^{k b} \alpha(t) d \beta(t) .
$$

\section{Prova}

Por hipótese, $f \in K^{g}([a, b], L(W, X))$.

Portanto, $\forall \varepsilon>0, \exists \delta$ gauge de $[a . b]$ tal que $\forall D \in T D_{[a, b]} \delta$-fina temos que

$$
\left\|\sum_{i=1}^{|D|}\left\{f\left(\xi_{i}\right)\left[g\left(t_{i}\right)-g\left(t_{i-1}\right)\right]-\int_{t_{i-1}}^{K t_{i}} f(t) d g(t)\right\}\right\|<\varepsilon .
$$

Tomando somas aproximadas de ${ }^{k \int_{a}^{b}} a(t) f(t) d g(t)$ e $\operatorname{de}^{k \int_{a}^{b}} \alpha(t) d \beta(t)$ vem:

$$
\left\|\sum_{i=1}^{|D|} a\left(\xi_{i}\right) f\left(\xi_{i}\right)\left[g\left(t_{i}\right)-g\left(t_{i-1}\right)\right]-\sum_{i=1}^{|D|} \alpha\left(\xi_{i}\right)\left[f\left(t_{i}\right)-f\left(t_{i-1}\right)\right]\right\|=
$$




$$
=\left\|\sum_{i=1}^{|D|} \alpha\left(\xi_{i}\right)\left\{f\left(\xi_{i}\right)\left[g\left(t_{i}\right)-g\left(t_{i-1}\right)\right]-\int_{t_{i-1}}^{K_{i}} f(t) d g(t)\right\}\right\|=I .
$$

Se $\alpha_{i} \in L(X, Y)$ e $x_{i} \in X$, entào temos que:

$$
\sum_{i=1}^{n} \alpha_{i} x_{i}=\sum_{j=1}^{n}\left(\alpha_{j}-\alpha_{j-1}\right)\left(\sum_{i=j}^{n} x_{i}\right)+\alpha_{0}\left(\sum_{i=1}^{n} x_{i}\right) .
$$

Fazendo $\alpha_{i}=\alpha\left(\xi_{i}\right), \alpha_{0}=\alpha(a)$ e $x_{i}=f\left(\xi_{i}\right)\left[g\left(t_{i}\right)-g\left(t_{i-1}\right)\right]-{ }_{t_{i-1}}^{t_{i}} f(t) d g(t)$ temos, pelo LEMA DE SACKS-HENSTOCK (Lema 2.1.6), que

$$
\left\|\sum_{i=j}^{|D|} x_{i}\right\|<\varepsilon, \forall j .
$$

Dai,

$$
I=\left\|\sum_{j=1}^{|D|}\left[\alpha\left(\xi_{j}\right)-\alpha\left(\xi_{j-1}\right)\right]\left(\sum_{i=j}^{|D|} x_{i}\right)+\alpha(a) \sum_{i=1}^{|D|} x_{i}\right\| \leq S V(\alpha) \cdot \varepsilon+\|\alpha(a)\| \cdot \varepsilon
$$

e segue a tese.

Corolário 1 Sejam a $\in S V([a, b], L(X, Y)), g \in \mathcal{C}([a, b], W), f \in K^{g}([a, b], L(W, X)) e$ $\beta=\dot{f}_{g}:[a, b] \rightarrow X$ Entâo $a \in R^{\beta}([a, b], L(X, Y)), \alpha \cdot f \in K^{g}([a, b], L(W, Y)) e$

$$
\int_{a}^{r i b} a(t) f(t) d g(t)=\int_{a}^{b} \alpha(t) d \beta(t) .
$$

Prova ( $\beta$ é contínua pelo corolário do Teorema 2.1.9 e daí, aplica-se o corolário do Teorema 1.4.1 item (ii)).

Outro resultado para a fórmula de integração por partes para integrais de Kurzweil é o seguinte: 
Teorema 2.1.15 Sejam $\alpha \in K([a, b], L(X, Y))$ e $f \in B V([a, b], X)$. Então $\alpha \cdot f \in K([a, b], Y)$ e vale

$$
\int_{a}^{k b} \alpha(t) f(t)=\int_{a}^{b} d \tilde{\alpha}(t) f(t)=\tilde{\alpha}(b) f(b)-\tilde{\alpha}(a) f(a)-\int_{a}^{b} \tilde{\alpha}(t) d f(t) .
$$

\section{Prova}

Por hipótese, $\alpha \in K([a, b], L(X, Y))$, então $\tilde{\alpha}$ é contínua (corolário do Teorema 2.1.9).

Além disso, $f \in B V([a, b], X)$.

Portanto $f \in R^{\dot{\alpha}}([a, b], X)$ pelo corolário do Teorema 1.4.1.

Então, como $\alpha \in K^{\prime}\left([a, b], L\left(X, Y^{\prime}\right)\right)$ e $f \in R^{\tilde{\alpha}}([a, b], X)$ temos que, $\forall \varepsilon>0, \exists \delta$ gauge de $[a, b]$ tal que $\forall D=\left(\xi_{i}, t_{i}\right) \in T D_{[a, b]}$ temos que

$$
\left\|\sum_{i=1}^{|D|}\left\{\alpha\left(\xi_{i}\right)\left(t_{i}-t_{i-1}\right)-\int_{t_{i}}^{k_{i-1}} \alpha(t) d t\right\}\right\| \leq \frac{\varepsilon}{2}
$$

$\mathrm{e}$

$$
\left\|\sum_{i=1}^{|D|}\left[\dot{\alpha}\left(t_{i}\right)-\dot{\alpha}\left(t_{i-1}\right)\right] f\left(\xi_{i}\right)-\int_{a}^{b} d \tilde{\alpha}(t) f(t)\right\| \leq \frac{\varepsilon}{2} .
$$

Então,

$$
\begin{gathered}
\left\|\sum_{i=1}^{|D|} \alpha\left(\xi_{i}\right) f\left(\xi_{i}\right)\left(t_{i}-t_{i-1}\right)-\int_{a}^{b} d \tilde{\alpha}(t) f(t)\right\| \leq \\
\leq\left\|\sum_{i=1}^{|D|} \alpha\left(\xi_{i}\right) f\left(\xi_{i}\right)\left(t_{i}-t_{i-1}\right)-\sum_{i=1}^{|D|}\left[\tilde{\alpha}\left(t_{i}\right)-\tilde{\alpha}\left(t_{i-1}\right)\right] f\left(\xi_{i}\right)\right\|+ \\
+\left\|\sum_{i=1}^{|D|}\left[\hat{\alpha}\left(t_{i}\right)-\dot{\alpha}\left(t_{i-1}\right)\right] f\left(\xi_{i}\right)-\int_{a}^{b} d \tilde{\alpha}(t) f(t)\right\| \leq \\
\leq\left\|\sum_{i=1}^{|D|}\left\{\alpha\left(\xi_{i}\right)\left(t_{i}-t_{i-1}\right)-\int_{t_{i}}^{K_{i}} \alpha(t) d t\right\} f\left(\xi_{i}\right)\right\|+\frac{\varepsilon}{2}=I .
\end{gathered}
$$

Para $\alpha_{i} \in L(X, Y)$ e $x_{i} \in \mathbb{X}$ temos que

$$
\sum_{i=1}^{n} \alpha_{i} x_{i}=\left(\sum_{i=1}^{n} \alpha_{i}\right) x_{0}+\sum_{j=1}^{n}\left(\sum_{i=j}^{n} \alpha_{j}\right)\left(x_{j}-x_{j-1}\right) .
$$


Então, fazendo $x_{i}=f\left(\xi_{i}\right), x_{0}=f(a)$ e $\alpha_{i}=\alpha\left(\xi_{i}\right)\left(t_{i}-t_{i-1}\right)-{ }^{k} \int_{t_{i-1}}^{t_{i}} \alpha(t) d t$ temos que

$$
\left\|\sum_{i=j}^{|D|} \alpha_{i}\right\| \leq \varepsilon, \forall j
$$

pelo LEMA DE SACKS-HENSTOCK (Lema 2.1.6).

E segue que

$$
\begin{gathered}
I \leq\left\|\sum_{i=1}^{|D|}\left\{\alpha\left(\xi_{i}\right)\left(t_{i}-t_{i-1}\right)-\int_{t_{i-1}}^{t_{i}} \alpha(t) d t\right\}\right\|\|f(a)\|+ \\
+\sum_{j=1}^{|D|}\left\|\sum_{i=j}^{|D|}\left\{\alpha\left(\xi_{i}\right)\left(t_{i}-t_{i-1}\right)-\int_{t_{i-1}}^{k_{i}} \alpha(t) d t\right\}\right\|\left\|f\left(\xi_{j}\right)-f\left(\xi_{j-1}\right)\right\|+\frac{\varepsilon}{2} \leq \\
\leq \frac{\varepsilon}{2} \cdot\|f(a)\|+\frac{\varepsilon}{2} \cdot V(f)+\frac{\varepsilon}{2} .
\end{gathered}
$$

Daí, de modo mais geral vem o teorema abaixo sobre a fórmula de substituição:

Teorema 2.1.16 Sejam $\alpha:[a, b] \rightarrow L(X, Y), \alpha \in K^{\alpha}([a, b], L(W, X))$ e sejam também $\beta=\hat{\alpha}_{\alpha}:[a, b] \rightarrow L(W, Y)$ ef $f \in B V([a, b], W)$. Então $\alpha \cdot f \in K^{\alpha}([a, b], X)$ se e só se $f \in K^{\prime \beta}([a, b], W)$. Neste caso,

$$
\int_{a}^{k b} d a(t) \alpha(t) f(t)=\int_{a}^{k b} d \beta(t) f(t) .
$$

\section{Prova}

Por hipótese, $a \in K^{a}([a, b], L(W, X))$.

Então $\forall \varepsilon>0, \exists \delta \mathrm{um}$ gauge de $[a, b]$ tal que $\forall D=\left(\xi_{i}, t_{i}\right) \in T D_{[a, b]}$ temos que:

$$
\left\|\sum_{i=1}^{|D|}\left\{\left[\alpha\left(t_{i}\right)-\alpha\left(t_{i-1}\right)\right] \alpha\left(\xi_{i}\right)-\int_{t_{i-1}}^{t_{i}} d \alpha(t) \alpha(t)\right\}\right\|<\varepsilon .
$$


Tomando somas aproximadas para as integrais ${ }^{k} \int_{a}^{b} d \alpha(t) \alpha(t) f(t) \mathrm{e}^{K^{b}} \int_{a}^{b} d \beta(t) f(t)$, temos:

$$
\begin{aligned}
& \left\|\sum_{i=1}^{|D|}\left[\alpha\left(t_{i}\right)-\alpha\left(t_{i-1}\right)\right] \alpha\left(\xi_{i}\right) f\left(\xi_{i}\right)-\sum_{i=1}^{|D|}\left[\beta\left(t_{i}\right)-\beta\left(t_{i-1}\right)\right] f\left(\xi_{i}\right)\right\|= \\
& \quad=\left\|\sum_{i=1}^{|D|}\left\{\left[\alpha\left(t_{i}\right)-\alpha\left(t_{i-1}\right)\right] \alpha\left(\xi_{i}\right)-\int_{t_{i-1}}^{K_{i}} d \alpha(t) \alpha(t)\right\} f\left(\xi_{i}\right)\right\| .
\end{aligned}
$$

Se $\alpha_{i} \in L(W, Y)$ e $x_{i} \in W$, temos:

$$
\sum_{i=1}^{n} \alpha_{i} x_{i}=\left(\sum_{i=1}^{n} \alpha_{i}\right) x_{0}+\sum_{j=1}^{n}\left(\sum_{i=j}^{n} \alpha_{j}\right)\left(x_{j}-x_{j-1}\right) .
$$

Seja

$$
I=\left\|\sum_{i=1}^{|D|}\left\{\left[\alpha\left(t_{i}\right)-\alpha\left(t_{i-1}\right)\right] \alpha\left(\xi_{i}\right)-\int_{t_{i-1}}^{K_{i}} d \alpha(t) \alpha(t)\right\} f\left(\xi_{i}\right)\right\| .
$$

Entào, fazendo $x_{i}=f\left(\xi_{i}\right), x_{0}=f(a)$ e $\alpha_{i}=\left[\alpha\left(t_{i}\right)-\alpha\left(t_{i-1}\right)\right] \alpha\left(\xi_{i}\right)-{ }^{k} \int_{t_{i-1}}^{t_{i}} d \alpha(t) \alpha(t)$, temos:

$$
\left\|\sum_{i=j}^{|D|} \alpha_{i}\right\| \leq \varepsilon, \forall j
$$

pelo LEMA DE SACKS-HENSTOCK (Lema 2.1.6).

Daí,

$$
\begin{gathered}
I \leq\left\|\sum_{i=1}^{|D|}\left\{\left[\alpha\left(t_{i}\right)-\alpha\left(t_{i-1}\right)\right] \alpha\left(\xi_{i}\right)-\int_{t_{i-1}}^{t_{i}} d \alpha(t) \alpha(t)\right\}\right\|\|f(a)\|+ \\
+\sum_{j=1}^{|D|}\left\|\sum_{i=j}^{|D|}\left\{\left[\alpha\left(t_{i}\right)-\alpha\left(t_{i-1}\right)\right] \alpha\left(\xi_{i}\right)-\int_{t_{i-1}}^{K t_{i}} d \alpha(t) \alpha(t)\right\}\right\|\left\|f\left(\xi_{j}\right)-f\left(\xi_{j-1}\right)\right\|< \\
<\varepsilon \cdot\|f(a)\|+\varepsilon \cdot V(f),
\end{gathered}
$$

e segue a tese.

Com um exemplo análogo ao Exemplo 3 anterior, podemos mostrar que a fórmula de substituição do teorema anterior pode nào ser verdadeira se não fizermos a exigência de que $f$ seja de variaçào limitada. 
Corolário 1 Sejam $\gamma \in \mathcal{C}([a, b], L(X, Y)), \alpha \in K^{\gamma}([a, b], L(W, X)), \beta=\tilde{\alpha}_{\gamma}:[a, b] \rightarrow$ $L(W, Y)$ e $f \in B V([a, b], W)$. Então $g \in R^{\beta}([a, b], W)$, alpha $f \in K^{\gamma}([a, b], X) e$

$$
\int_{a}^{\kappa^{b}} d \gamma(t) \alpha(t) f(t)=\int_{a}^{b} d \beta(t) f(t) .
$$




\subsection{A Integral vetorial de Henstock}

Sejam $f:[a, b] \rightarrow X$ e $a:[a, b] \rightarrow L(X, Y)$

\section{Definição 2.2.1 Dizemos que:}

(i) $f$ é Henstock integrável em relação à $\alpha$, e escrevemos $f \in H^{\alpha}([a, b], X)$, se existe uma função $F_{\alpha}:[a, b] \rightarrow Y$ satisfazendo:

$$
\begin{gathered}
\forall \varepsilon>0, \exists \delta \text { gauge de }[a, b] \text { tal que } \forall D=\left(\xi_{i}, t_{i}\right) \in T D_{[a, b]} \delta \text {-fina temos que } \\
\sum_{i=1}^{|D|}\left\|F_{\alpha}\left(t_{i}\right)-F_{\alpha}\left(t_{i-1}\right)-\left[\alpha\left(t_{i}\right)-\alpha\left(t_{i-1}\right)\right] f\left(\xi_{i}\right)\right\|<\varepsilon .
\end{gathered}
$$

Neste caso, dizemos que $F_{\mathrm{o}}$ é associada à $f(q u a n d o ~ \alpha(t)=t$, escrevemos $H([a, b], X)$ ).

(ii) $\alpha$ é Henstock integrável em relação à $f$, e escrevemos $f \in H^{f}([a, b], L(X, Y))$, se existe uma funçào $A_{f}:[a, b] \rightarrow Y$ satisfazendo:

$$
\begin{aligned}
\forall \varepsilon>0, \exists \delta \text { gauge de }[a, b] \text { tal que } \forall D=\left(\xi_{i}, t_{i}\right) \in T D_{[a, b]} \delta \text {-fina temos que } \\
\\
\sum_{i=1}^{|D|}\left\|A_{f}\left(t_{i}\right)-A_{f}\left(t_{i-1}\right)-\alpha\left(\xi_{i}\right)\left[f\left(t_{i}\right)-f\left(t_{i-1}\right)\right]\right\|<\varepsilon .
\end{aligned}
$$

Neste caso, dizemos que $A_{f}$ é associada à $\alpha$.

Com a notaçào da definiçào acima. vemos que $f \in K^{\alpha}\left(\left[t_{i-1}, t_{i}\right], X\right) \mathrm{e}^{K_{t_{i-1}}^{t_{i}}} d \alpha(t) f(t)=$ $F_{\alpha}\left(t_{i}\right)-F_{\alpha}\left(t_{i-1}\right) . \forall i$. Entào, $f \in K^{\circ}([a, b], X) \mathrm{e}^{K_{0}^{b}} d \alpha(t) f(t)=F_{\alpha}(b)-F_{\alpha}(a)$. Do mesmo

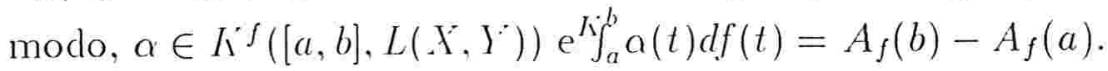

Assim, podemos enunciar o 


\section{Teorema 2.2.2 Temos:}

(i) $H^{\alpha}([a, b], X) \subset K^{\alpha}([a, b], X)$ e, se $f \in H^{\alpha}([a, b], X)$ com $F_{\alpha}$ associada à $f$, então $K_{\int_{a}^{b}}^{b} d \alpha(t) f(t)=F_{\alpha}(b)-F_{\alpha}(a)$.

(ii) $H^{f}([a, b], L(X, Y)) \subset K^{f}([a, b], L(X, Y))$ e, se $\alpha \in H^{f}([a, b], L(X, Y))$ com $A_{f}$ associada à $\alpha$, então ${ }^{K \int_{a}^{b}} \alpha(t) d f(t)=A_{f}(b)-A_{f}(a)$.

Podemos ter $R([a, b], X) \not \subset H([a, b], X)$. De fato,

Exemplo 4 Sejam $X=l_{2}([a, b])$ e $f:[a, b] \rightarrow X$ definida por $f(t)=e_{t}$.

Já vimos que $f \in R([a, b], X) \operatorname{com} \tilde{f}(\xi)=0, \forall \xi \in[a, b]$.

Sejam $x_{0}, x \in[a, b], \operatorname{com} x_{0} \leq x$.

Suponhamos, por absurdo, que $f \in H([a, b], X)$ e seja $F$ associada à $f$.

Entâo $F(x)-F\left(x_{0}\right)=\int_{x_{0}}^{x} f(t) d t=\hat{f}(x)-\hat{f}\left(x_{0}\right)=0, \forall\left[x_{o}, x\right] \subset[a, b]$.

Portanto Fé constante.

Logo, $\forall D=\left(\xi_{i}, t_{i}\right) \in T D_{[a, b]}$ temos que

$$
\sum_{i=1}^{|D|}\left\|F\left(t_{i}\right)-F\left(t_{i-1}\right)-f\left(\xi_{i}\right)\left(t_{i}-t_{i-1}\right)\right\|=\sum_{i=1}^{|D|}\left\|e_{\xi_{i}}\left(t_{i}-t_{i-1}\right)\right\|=\sum_{i=1}^{|D|}\left|t_{i}-t_{i-1}\right|=b-a,
$$

uma contradoçào.

$A \operatorname{sim}, f \notin H([a, b] . X)$.

Mais geralmente, quando a dimensào de $X$ é infinita podemos ter $R^{\alpha}([a, b], X) \not \subset H^{\alpha}([a, b], X)$, $R^{f}\left([a, b], L(X . Y) \not \subset H^{f}([a, b], L(X, Y)), K^{\alpha}([a, b], X) \not \subset H^{\alpha}([a, b], X)\right.$ e $K^{f}([a, b], L(X . Y)) \not \subset$ $H^{f}([a, b], L(X, Y))$.

Nos espaços $H^{\alpha}\left([a, b], X^{*}\right)$ e $H^{f}([a, b], L(X, Y))$ valem as seguintes propriedades:

(1) bilinearidacle da integral:

(2) integrabilidade num intervalo implica integrabilidade nos subintervalos e vice-versa. 
Lema 2.2.3 (LEMA DE SACKS-HENSTOCK)

(i) Seja $f \in H^{\alpha}([a, b], X)$.

Se para $\varepsilon>0$, o gauge $\delta$ de $[a, b]$ é tal que $\forall D=\left(\xi_{i}, t_{i}\right) \in T D_{[a, b]} \delta$-fina temos

$$
\sum_{i=1}^{|D|}\left\|\int_{t_{i-1}}^{K_{i}} d \alpha(t) f(t)-\left[\alpha\left(t_{i}\right)-\alpha\left(t_{i-1}\right)\right] f\left(\xi_{i}\right)\right\|<\varepsilon,
$$

entầ, para $a \leq c_{1} \leq \eta_{1} \leq d_{1} \leq c_{2} \leq \eta_{2} \leq d_{2} \leq \ldots \leq c_{k} \leq \eta_{k} \leq d_{k} \leq b$ com $\eta_{j} \in\left[c_{j}, d_{j}\right] \subset B_{\delta\left(\eta_{j}\right)}\left(\eta_{j}\right)$ para cada $j$, temos que

$$
\sum_{j=1}^{k}\left\|\int_{c_{j}}^{K d_{j}} d \alpha(t) f(t)-\left[\alpha\left(d_{j}\right)-\alpha\left(c_{j}\right)\right] f\left(\eta_{j}\right)\right\|<\varepsilon ;
$$

(ii) Seja $\alpha \in H^{f}([a, b], L(X, Y))$.

Se para $\Xi>0$. o gauge $\delta$ de $[a, b]$ é tal que $\forall D=\left(\xi_{i}, t_{i}\right) \in T D_{[a, b]} \delta$-fina temos

$$
\sum_{i=1}^{|D|}\left\|\int_{t_{i-1}}^{I t_{i}} a(t) d f(t)-\alpha\left(\xi_{i}\right)\left[f\left(t_{i}\right)-f\left(t_{i-1}\right)\right]\right\|<\varepsilon,
$$

entâo, para $a \leq c_{1} \leq \eta_{1} \leq d_{1} \leq c_{2} \leq \eta_{2} \leq d_{2} \leq \ldots \leq c_{k} \leq \eta_{k} \leq d_{k} \leq b$ com $\eta_{j} \in\left[c_{j}, d_{j}\right] \subset B_{\delta\left(\eta_{j}\right)}\left(\eta_{j}\right)$ para cada $j$, temos que

$$
\sum_{j=1}^{k}\left\|\int_{c_{j}}^{k d_{j}} \alpha(t) d f(t)-\alpha\left(\eta_{j}\right)\left[f\left(d_{j}\right)-f\left(c_{j}\right)\right]\right\|<\varepsilon .
$$

Se $f \in H^{\alpha}([a, b], X)$. entào $f \in K^{\circ}([a, b], X)$ pelo Teorema 2.2.2. Daí, segue que existe $\dot{f}_{\alpha}:[a, b] \rightarrow Y$ definida por $\hat{f}_{\alpha}(\xi)={ }^{k} \int_{a}^{\xi} d \alpha(t) f(t)$ e, sendo $F_{\alpha}$ associada à $f$, temos que $\tilde{f}_{\alpha}(\xi)=$ $F_{\alpha}(\xi)-F_{\alpha}(a), \forall \xi \in[a, b]$, também pelo Teorema 2.2.2. Analogamente, temos que $\tilde{\alpha}_{f}(\xi)=$ $A_{f}(\xi)-A_{f}(a), \forall \xi \in[a, b]$ para $\alpha \in H^{f}([a, b], L(X, Y))$ com $A_{f}$ associada à $\alpha$.

Daí, pelos Teoremas 2.1.9 e 2.1.10 seguem, respectivamente, os Teoremas 2.2.4 e 2.2.5. 
Teorema 2.2.4 Sejam $f \in G([a, b], X), \alpha \in H^{f}([a, b], L(X, Y))$ e $A_{f}$ associada à $\alpha$. Então $A_{f} \in G([a, b], Y) \mathrm{com}$

$$
A_{f}(\xi+)-A_{f}(\xi)=\alpha(\xi)[f(\xi+)-f(\xi)], \forall \xi \in[a, b[
$$

$e$

$$
\left.\left.A_{f}(\xi)-A_{f}(\xi-)=\alpha(\xi)[f(\xi)-f(\xi-)], \forall \xi \in\right] a, b\right]
$$

Corolário 1 Se $f \in \mathcal{C}([a, b], X)$ e $\alpha \in H^{f}([a, b], L(X, Y))$, então $A_{f}$ associada à $\alpha$ é tal que $A_{f} \in \mathcal{C}([a, b], Y)$.

Teorema 2.2.5 Sejam $\alpha \in G^{\sigma}([a, b], L(X, Y))$ e $f \in H^{\alpha}([a, b], X)$ com $F_{\alpha}$ associada à $f$. Entâo $F_{a} \in G([a, b], Y)$ com

$$
F_{\alpha}(\xi+)-F_{\alpha}(\xi)=[\alpha(\xi \dot{+})-\alpha(\xi)] f(\xi), \forall \xi \in[a, b[
$$

$\epsilon$

$$
\left.\left.F_{\mathrm{o}}(\xi)-F_{\mathrm{o}}(\xi-)=[\alpha(\xi)-\alpha(\xi \dot{-})] f(\xi), \forall \xi \in\right] a, b\right]
$$

Corolário 1 Se $a \in \mathcal{C}^{\sigma}\left([a, b] . L\left(X, Y^{*}\right)\right) \in f \in H^{\alpha}([a, b], X)$, então $F_{\alpha}$ associada à $f$ é tal que $F_{\alpha} \in \mathcal{C}\left([a, b], Y^{\prime}\right)$.

O teorema seguinte dá um resultado sobre a fórmula de integração por partes para a integral de Henstock.

Teorema 2.2.6 Se $a \in B V([a . b] . L(X . Y))$ e $f \in H([a, b], X)$, entâo $\alpha \cdot f \in H([a, b], Y)$ e

$$
\int_{a}^{k b} \alpha(t) f(t) d t=\int_{a}^{b} a(t) d \tilde{f}(t)=\alpha(b) \tilde{f}(b)-\alpha(a) \tilde{f}(a)-\int_{a}^{b} d a(t) \tilde{f}(t) .
$$




\section{Prova}

Por hipótese, $f \in H([a, b], X)$.

Entã.o, $\forall \varepsilon>0, \exists \delta$ um gauge de $[a, b]$ tal que $\forall D=\left(\xi_{i}, t_{i}\right) \in T D_{[a, b]}$ temos que

$$
\sum_{i=1}^{|D|}\left\|f\left(\xi_{i}\right)\left(t_{i}-t_{i-1}\right)-\int_{t_{i-1}}^{t_{i}} f(t) d t\right\|<\varepsilon .
$$

Portanto,

$$
\begin{gathered}
\sum_{i=1}^{|D|}\left\|\alpha\left(\xi_{i}\right) f\left(\xi_{i}\right)\left(t_{i}-t_{i-1}\right)-\int_{t_{i-1}}^{K} \alpha(t) f(t) d t\right\| \leq \\
\leq \sum_{i=1}^{t_{i}}\left\|\alpha\left(\xi_{i}\right)\left\{f\left(\xi_{i}\right)\left(t_{i}-t_{i-1}\right)-\int_{t_{i-1}}^{\xi_{i}} f(t) d t\right\}\right\|+\sum_{i=1}^{|D|}\left\|\int_{t_{i-1}}^{K t_{i}}\left[\alpha(t)-\alpha\left(\xi_{i}\right)\right] f(t) d t\right\|< \\
<\|\alpha\| \cdot \varepsilon+\sum_{i=1}^{|D|}\left\|\int_{t_{i-1}}^{K t_{i}}\left[\alpha(t)-\alpha\left(\xi_{i}\right)\right] f(t) d t\right\| .
\end{gathered}
$$

Mas, pelos Teoremas 2.2 .2 e 2.1.13, temos que $\alpha \cdot f \in K([a, b], X)$ e

$$
\int_{a}^{k b} \alpha(t) f(t) d t=\int_{a}^{b} \alpha(t) d \hat{f}(t)=\alpha(b) \tilde{f}(b)-\alpha(a) \tilde{f}(a)-\int_{a}^{b} d \alpha(t) \tilde{f}(t) .
$$

Logo,

$$
\begin{gathered}
\sum_{i=1}^{|D|}\left\|\int_{t_{1-1}}^{K \hat{t}_{i}}\left[\alpha(t)-\alpha\left(\xi_{i}\right)\right] f(t) d t\right\|= \\
=\sum_{i=1}^{|D|}\left\|\left[\alpha\left(t_{i}\right)-\alpha\left(\xi_{i}\right)\right] \dot{f}\left(t_{i}\right)-\left[\alpha\left(t_{i-1}\right)-\alpha\left(\xi_{i}\right)\right] \tilde{f}\left(t_{i-1}\right)-\int_{t_{i-1}}^{t_{i}} d \alpha(t) \tilde{f}(t)\right\|= \\
=\sum_{i=1}^{|D|}\left\|\left[\alpha\left(t_{i}\right)-\alpha\left(\xi_{i}\right)\right] \dot{f}\left(t_{i}\right)-\int_{\xi_{i}}^{t_{i}} d \alpha(t) \hat{f}(t)+\left[\alpha\left(\xi_{i}\right)-\alpha\left(t_{i-1}\right)\right] \tilde{f}\left(t_{i-1}\right)-\int_{t_{i-1}}^{\xi_{i}} d \alpha(t) \tilde{f}(t)\right\|= \\
=\sum_{i=1}^{|D|}\left\|\int_{\xi_{i}}^{t_{1}} d \alpha(t)\left[\hat{f}\left(t_{i}\right)-\dot{f}(t)\right]+\int_{t_{1-1}}^{\xi_{i}} d \alpha(t)\left[\tilde{f}\left(t_{i-1}\right)-\tilde{f}(t)\right]\right\| \leq V(\alpha) \cdot \omega(\tilde{f}) .
\end{gathered}
$$

E, pelo corolário do Teorema 2.2.5. temos que $\tilde{f} \in \mathcal{C}([a, b], X)$ e segue a tese. 
Mais geralmente temos o seguinte teorema para a fórmula de substituição:

Teorema 2.2.7 Sejam $\alpha:[a, b] \rightarrow L(X, Y)$ limitada, $g:[a, b] \rightarrow W, f \in H^{g}([a, b], L(W, X))$ e $\beta=\dot{f}_{g}:[a, b] \rightarrow X$. Então, $\alpha \in H^{\beta}([a, b], L(X, Y))$ se e só se $\alpha \cdot f \in H^{g}([a, b], Y)$. Neste caso,

$$
\int_{a}^{k b} \alpha(t) f(t) d g(t)=\int_{a}^{k b} \alpha(t) d \beta(t) .
$$

\section{Prova}

$(\Longrightarrow)$ Por hipótese, $f \in H^{g}([a, b], L(W, X))$ e $\alpha \in H^{\beta}([a, b], L(X, Y))$ com $\alpha$ limitada.

Portanto, $\forall \Xi>0, \exists \delta$ gauge de $[a, b]$ tal que $\forall D=\left(\xi_{i}, t_{i}\right) \in T D_{[a, b]} \delta$-fina temos que:

$$
\sum_{i=1}^{|D|}\left\|f(\xi)\left[g\left(t_{i}\right)-g\left(t_{i-1}\right)\right]-\int_{t_{i-1}}^{K_{i} t_{i}} f(t) d g(t)\right\|<\frac{\varepsilon}{2 \cdot\|\alpha\|}
$$

e

$$
\sum_{i=1}^{|D|}\left\|\alpha(\xi)\left[\beta\left(t_{i}\right)-\beta\left(t_{i-1}\right)\right]-\int_{t_{i-1}}^{K_{i}} \alpha(t) d \beta(t)\right\|<\frac{\varepsilon}{2} .
$$

Entào.

$$
\begin{gathered}
\sum_{i=1}^{|D|}\left\|\alpha(\xi) f(\xi)\left[g\left(t_{i}\right)-g\left(t_{i-1}\right)\right]-\int_{t_{i-1}}^{K t_{i}} \alpha(t) d \beta(t)\right\| \leq \\
\leq \sum_{i=1}^{|D|}\left\|\alpha(\xi) f(\xi)\left[g\left(t_{i}\right)-g\left(t_{i-1}\right)\right]-\alpha(\xi)\left[\beta\left(t_{i}\right)-\beta\left(t_{i-1}\right)\right]\right\|+ \\
+\sum_{i=1}^{|D|}\left\|\alpha(\xi)\left[\beta\left(t_{i}\right)-\beta\left(t_{i-1}\right)\right]-\int_{t_{i-1}}^{K t_{i}} \alpha(t) d \beta(t)\right\| \leq \\
\leq\|\alpha\| \sum_{i=1}^{|D|}\left\|f(\xi)\left[g\left(t_{i}\right)-g\left(t_{i-1}\right)\right]-\int_{t_{i-1}}^{K t_{i}} f(t) d g(t)\right\|+\frac{\varepsilon}{2}<\|\alpha\| \cdot \frac{\varepsilon}{2 \cdot\|\alpha\|}+\frac{\varepsilon}{2}=\varepsilon .
\end{gathered}
$$

$(\Longleftarrow)$ Por hipótese, $f \in H^{g}([a, b], L(W, X))$ e $\alpha \cdot f \in H^{g}([a, b], Y)$ com $\alpha$ limitada. 
Portanto, $\forall \varepsilon>0, \exists \delta$ gauge de $[a, b]$ tal que $\forall D=\left(\xi_{i}, t_{i}\right) \in T D_{[a, b]} \delta$-fina temos que:

$$
\sum_{i=1}^{|D|}\left\|f(\xi)\left[g\left(t_{i}\right)-g\left(t_{i-1}\right)\right]-\int_{t_{i-1}}^{K_{i}} f(t) d g(t)\right\|<\frac{\varepsilon}{2 \cdot\|\alpha\|}
$$

$\mathrm{e}$

$$
\sum_{i=1}^{|D|}\left\|\alpha(\xi) f\left(\xi_{i}\right)\left[g\left(t_{i}\right)-g\left(t_{i-1}\right)\right]-\int_{t_{i-1}}^{K_{i}} \alpha(t) f(t) d g(t)\right\|<\frac{\varepsilon}{2} .
$$

Então,

$$
\begin{gathered}
\sum_{i=1}^{|D|}\left\|\alpha(\xi)\left[\beta\left(t_{i}\right)-\beta\left(t_{i-1}\right)\right]-\int_{t_{i-1}}^{K t_{i}} \alpha(t) f(t) d g(t)\right\| \leq \\
\leq \sum_{i=1}^{|D|}\left\|\alpha(\xi)\left[\beta\left(t_{i}\right)-\beta\left(t_{i-1}\right)\right]-\alpha(\xi) f(\xi)\left[g\left(t_{i}\right)-g\left(t_{i-1}\right)\right]\right\|+ \\
+\sum_{i=1}^{|D|}\left\|\alpha(\xi) f(\xi)\left[g\left(t_{i}\right)-g\left(t_{i-1}\right)\right]-\int_{t_{i-1}}^{K t_{i}} \alpha(t) f(t) d g(t)\right\| \leq \\
\leq\|\alpha\| \sum_{i=1}^{|D|}\left\|\int_{t_{t_{-}}}^{K \dot{t}_{i}} f(t) \operatorname{dg}(t)-f(\xi)\left[g\left(t_{i}\right)-g\left(t_{i-1}\right)\right]\right\|+\frac{\varepsilon}{2}<\|\alpha\| \cdot \frac{\varepsilon}{2 \cdot\|\alpha\|}+\frac{\varepsilon}{2}=\varepsilon .
\end{gathered}
$$

Como caso particular temos o

Corolário 1 Sejam $f \in H([a, b], X)$ e $\alpha:[a, b] \rightarrow L(X, Y)$ limitada. Então temos que $\alpha \in H^{j}\left([a, b], L\left(X^{\prime} . Y^{*}\right)\right)$ se e só se $\alpha \cdot f \in H([a, b], Y)$ e

$$
\int_{a}^{k i p} \alpha(t) f(t) d t=\int_{a}^{k p} \alpha(t) d \tilde{f}(t) .
$$

Segue agora outro resultado para a fórmula de integração por partes para as integrais de Henstock.

Teorema 2.2.8 Se $a \in H([a, b] . L(X . Y))$ e $f \in B V([a, b], X)$, então $\alpha \cdot f \in H([a . b] . Y)$ e

$$
\int_{a}^{k-b} a(t) f(t) d t=\int_{a}^{b} d \dot{\alpha}(t) f(t)=\tilde{\alpha}(b) f(b)-\tilde{\alpha}(a) f(a)-\int_{a}^{b} \tilde{\alpha}(t) d f(t) .
$$


Prova (análoga à demonstraçào do Teorema 2.2.6).

E, de modo mais geral, temos o seguinte resultado para a fórmula de substituição:

Teorema 2.2.9 Sejam $\gamma:[a, b] \rightarrow L(X, Y), \alpha \in H^{\gamma}([a, b], L(W, X))$ e sejam também $\beta=\check{a}_{\gamma}:[a, b] \rightarrow L(W, Y)$ e $f:[a, b] \rightarrow W$ limitada. Então, $f \in H^{\beta}([a, b], W)$ se $e$ só se $a \cdot f \in H^{\gamma}([a, b], X)$. Neste caso,

$$
\int_{a}^{k \cdot b} d \gamma(t) \alpha(t) f(t)=\int_{a}^{k b} d \beta(t) f(t) .
$$

\section{Prova}

$(\Longrightarrow)$ Por hipótese. $a \in H^{\gamma}([a, b], L(W, X))$ e $f \in H^{\beta}([a, b], W)$.

Logo, $\forall \Xi>0, \exists \delta$ gauge de $[a, b]$ tal que $\forall D=\left(\xi_{i}, t_{i}\right) \in T D_{[a, b]} \delta$-fina temos que:

$$
\sum_{i=1}^{|D|}\left\|\left[\gamma\left(t_{i}\right)-\gamma\left(t_{i-1}\right)\right] \alpha\left(\xi_{i}\right)-\int_{t_{i-1}}^{K_{i}} d \gamma(t) \alpha(t)\right\|<\frac{\varepsilon}{2\|f\|}
$$

e

$$
\sum_{i=1}^{|D|}\left\|\left[\beta\left(t_{i}\right)-\beta\left(t_{i-1}\right)\right] f\left(\xi_{i}\right)-\int_{t_{i-1}}^{K_{i}} d \beta(t) f(t)\right\|<\frac{\varepsilon}{2} .
$$

Portanto.

$$
\begin{gathered}
\sum_{i=1}^{|D|}\left\|\left[\gamma\left(t_{i}\right)-\gamma\left(t_{i-1}\right)\right] \alpha\left(\xi_{i}\right) f\left(\xi_{i}\right)-\int_{t_{i-1}}^{K t_{i}} d \beta(t) f(t)\right\| \leq \\
\leq \sum_{i=1}^{|D|}\left\|\left[\gamma\left(t_{i}\right)-\gamma\left(t_{i-1}\right)\right] \alpha\left(\xi_{i}\right) f\left(\xi_{i}\right)-\left[\beta\left(t_{i}\right)-\beta\left(t_{i-1}\right)\right] f\left(\xi_{i}\right)\right\|+ \\
\quad+\sum_{i=1}^{|D|}\left\|\left[\beta\left(t_{i}\right)-\beta\left(t_{i-1}\right)\right] f\left(\xi_{i}\right)-\int_{t_{i-1}}^{K t_{i}} d \beta(t) f(t)\right\|< \\
<\sum_{i=1}^{|D|}\left\|\left[\gamma_{i}\left(t_{i}\right)-\gamma\left(t_{i-1}\right)\right] \alpha\left(\xi_{i}\right)-\int_{t_{1-1}}^{K t_{i}} d \gamma(t) \alpha(t)\right\|\left\|f\left(\xi_{i}\right)\right\|+\frac{\varepsilon}{2}<\varepsilon .
\end{gathered}
$$


$(\Longleftarrow)$ Por hipótese, $\alpha \in H^{\gamma}([a, b], L(W, X))$ e $\alpha \cdot f \in H^{\gamma}([a, b], Y)$.

Logo, $\forall \varepsilon>0, \exists \delta$ gauge de $[a, b]$ tal que $\forall D=\left(\xi_{i}, t_{i}\right) \in T D_{[a, b]} \delta$-fina temos que:

$$
\sum_{i=1}^{|D|}\left\|\left[\gamma\left(t_{i}\right)-\gamma\left(t_{i-1}\right)\right] \alpha\left(\xi_{i}\right)-\int_{t_{i-1}}^{K_{i}} d \gamma(t) \alpha(t)\right\|<\frac{\varepsilon}{2\|f\|}
$$

$\mathrm{e}$

$$
\sum_{i=1}^{|D|}\left\|\left[\gamma\left(t_{i}\right)-\gamma\left(t_{i-1}\right)\right] \alpha\left(\xi_{i}\right) f\left(\xi_{i}\right)-\int_{t_{i-1}}^{K_{i}} d \gamma(t) \alpha(t) f(t)\right\|<\frac{\varepsilon}{2}
$$

Portanto,

$$
\begin{gathered}
\sum_{i=1}^{|D|}\left\|\left[\beta\left(t_{i}\right)-\beta\left(t_{i-1}\right)\right] f\left(\xi_{i}\right)-\int_{t_{i-1}}^{K_{i}} d \gamma(t) \alpha(t) f(t)\right\| \leq \\
\leq \sum_{i=1}^{|D|}\left\|\left(\int_{t_{,-1}}^{K t_{i}} d \gamma(t) \alpha(t)\right) f\left(\xi_{i}\right)-\left[\gamma\left(t_{i}\right)-\gamma\left(t_{i-1}\right)\right] \alpha\left(\xi_{i}\right) f\left(\xi_{i}\right)\right\|+ \\
+\sum_{i=1}^{|D|}\left\|\left[\gamma\left(t_{i}\right)-\gamma\left(t_{i-1}\right)\right] \alpha\left(\xi_{i}\right) f\left(\xi_{i}\right)-\int_{t_{i-1}}^{K t_{i}} d \gamma(t) \alpha(t) f(t)\right\|< \\
<\sum_{i=1}^{|D|}\left\|\int_{t_{i-1}}^{K t_{i}} d \gamma(t) \alpha(t)-\left[\gamma\left(t_{i}\right)-\gamma\left(t_{i-1}\right)\right] \alpha\left(\xi_{i}\right)\right\|\left\|f\left(\xi_{i}\right)\right\|+\frac{\varepsilon}{2}<\varepsilon .
\end{gathered}
$$

Em particular vem o

Corolário 1 Sejam $\alpha \in H([a, b], L(X, Y))$ e $f:[a, b] \rightarrow X$ limitada. Entâo temos que $f \in H^{\dot{a}}([a, b], X)$ se $e$ só se $\alpha \cdot f \in H([a, b], Y)$ e

$$
\int_{a}^{K} \alpha(t) f(t) d t=\int_{a}^{k b} d \tilde{\alpha}(t) f(t) .
$$




\subsection{Integrais Vetoriais Impróprias}

Sejam $f:[a, b] \rightarrow X$ e $a:[a, b] \rightarrow L(X, Y)$

Teorema 2.3.1 Sejam $\alpha:[a, b] \rightarrow L(X, Y)$ fracamente contínua em a e $f \in K^{\alpha}([c, b], X)$, $\forall c \in] a, b]$. Entào $f \in K^{\alpha}([a, b], X)$ se e só se $\exists \lim _{c \downarrow a} \int_{c}^{b} d \alpha(t) f(t)$. Neste caso,

$$
\int_{a}^{k i b} d \alpha(t) f(t)=\lim _{c \downarrow a} \int_{c}^{k b} d \alpha(t) f(t) .
$$

\section{Prova}

$(\Longrightarrow)$ Pelo Teorema 2.1.10 e corolário vem que $\exists \tilde{f}_{\alpha}(a+)=\tilde{f}_{\alpha}(a)$ e acabou.

$(\Longleftarrow)$ Seja $\left(c_{n}\right)_{n \in N}$ uma sequência de elementos de $[a, b]$ tal que $c_{0}=b, c_{n}>c_{n+1}$ e $c_{n} \rightarrow a$.

Seja $\varepsilon>0$.

Portanto, para cada $n>0, \exists \delta_{n}$ gauge de $\left[c_{n}, c_{n-1}\right]$ que é especial relativamente à $c_{n}$ e $c_{n-1}$ tal que $\forall D_{n}=\left(\xi_{i_{n}}, t_{i_{n}}\right) \in T D_{\left[c_{n}, c_{n-1}\right]} \delta_{n}$-fina temos que

$$
\left\|\sum_{i_{n}=1}^{\left|D_{n}\right|}\left[\alpha\left(t_{i_{n}}\right)-\alpha\left(t_{i_{n}-1}\right)\right] f\left(\xi_{i_{n}}\right)-\int_{c_{n}}^{K c_{n-1}} d \alpha(t) f(t)\right\|<\frac{\varepsilon}{2^{n}} .
$$

Mas, $\alpha$ é fracamente contínua em $a$ e $\exists \lim _{c\rfloor a} \int_{c}^{b} d \alpha(t) f(t)=A$.

Logo, $\exists n_{0} \in I$ tal que para $a<c \leq c_{n_{0}}$ temos que

$$
\|[a(c)-a(a)] f(a)\|<\varepsilon \mathrm{e}\left\|\int_{c}^{b} d \alpha(t) f(t)-A\right\|<\varepsilon
$$


Tomemos um gauge $\delta$ de $[a, b]$ tal que $\delta(a)<c_{n_{0}}-a, \delta(\xi) \leq \delta_{n}(\xi)$, se $\xi \in\left[c_{n}, c_{n-1}\right]$ e tal que $\delta$ é especial relativamente à $a$.

Seja $D=\left(\xi_{i}, t_{i}\right) \in T D_{[a, b]} \delta$-fina.

Para cada $n>0$, seja $D_{n}=\left\{\left(\xi_{i}, t_{i}\right) ;\left[t_{i-1}, t_{i}\right] \subset\left[c_{n}, c_{n-1}\right]\right\} \subset D$ e seja também $J_{n}=$ $\left\{\left[t_{i-1}, t_{i}\right] ;\left[t_{i-1}, t_{i}\right] \subset\left[c_{n}, c_{n-1}\right]\right\}$.

Então $D_{n} \in T D_{J_{n}}$ e é $\delta_{n}$-fina, para cada $n>0$.

Seja $D_{n}=\left(\xi_{i_{n}}, t_{i_{n}}\right)$, para cada $n>0$.

Então o LEMA DE SACKS-HENSTOCK (Lema 2.1.6) implica que

$$
\left\|\sum_{i_{n}=1}^{\left|D J_{n}\right|}\left[\alpha\left(t_{i_{n}}\right)-\alpha\left(t_{i_{n}-1}\right)\right] f\left(\xi_{i_{n}}\right)-\int_{J_{n}}^{K} d \alpha(t) f(t)\right\|<\frac{\varepsilon}{2^{n}}, \forall n>0,
$$

pois $J_{n} \subset\left[c_{n}, c_{n-1}\right]$.

Portanto,

$$
\begin{gathered}
\left\|s^{\alpha}(f, D)-A\right\| \leq\left\|\left[\alpha\left(t_{1}\right)-\alpha(a)\right] f(a)\right\|+ \\
+\left\|\sum_{n=0}^{\infty}\left\{\sum_{i_{n}=1}^{\left|D_{n}\right|}\left[\alpha\left(t_{i_{n}}\right)-\alpha\left(t_{i_{n}-1}\right)\right] f\left(\xi_{i_{n}}\right)-\int_{J_{n}}^{K} d \alpha(t) f(t)\right\}\right\|+\left\|\int_{t_{1}}^{K} d \alpha(t) f(t)-A\right\|< \\
<\varepsilon+\sum_{n=0}^{\infty} \frac{\varepsilon}{2^{n}}+\varepsilon<3 \varepsilon .
\end{gathered}
$$

E segue que $A={ }^{k \cdot h} \int_{a}^{b} d a(t) f(t)$.

Corolário 1 Se $f \in K([c, b], X), \forall c \in] a, b]$ então $f \in K([a, b], X)$ se e só se $\exists \lim _{c \mid a}^{K j_{c}^{b}} \int_{c} f(t) d t$. Neste caso,

$$
\int_{a}^{k i b} f(t) d t=\lim _{c \downarrow a} \int_{c}^{k b} f(t) d t
$$

De modo análogo pode-se mostrar o 
Teorema 2.3.2 Sejam $f:[a, b] \rightarrow X$ continua em a e $\left.\left.\alpha \in K^{f}([c, b], L(X, Y)), \forall c \in\right] a, b\right]$. Entâo $\alpha \in K^{f}([a, b], L(X, Y))$ se e só se $\exists \lim _{c \downarrow a} \int_{c}^{b} \alpha(t) d f(t)$. Neste caso,

$$
\int_{a}^{k b} \alpha(t) d f(t)=\lim _{c \downarrow a} \int_{c}^{k p} \alpha(t) d f(t) .
$$

Também pode-se mostrar resultados análogos aos Teoremas 2.3.1 e 2.3.2 para a impropriedade à direita.

Daí, segue que as integrais vetoriais de Kurzweil contém as integrais vetoriais impróprias.

Com relaçào ao espaço das funçòes Henstock integráveis, podemos, de igual modo, mostrar que ele contém as integrais impróprias. Vamos demonstrar este resultado para impropriedades à esquerda. À direita, um resultado análogo pode ser provado. Então vejamos:

Teorema 2.3.3 Sejam a $:[a, b] \rightarrow L(X, Y)$ fracamente contínua em a e $f \in H^{\alpha}([c, b], X)$, $\forall c \in] a, b]$. Entrio $f \in H^{\alpha}([a, b], X)$ se e só se $\exists \lim _{c \downarrow a} \int_{c}^{b} d \alpha(t) f(t)$. Neste caso,

$$
\int_{a}^{k \cdot p} d \alpha(t) f(t)=\lim _{c\rfloor a} \int_{c}^{k b} d \alpha(t) f(t)
$$

\section{Prova}

$(\Longleftarrow)$ Pelo Teorema 2.1 .10 e corolário vem que $\exists \dot{f}_{\alpha}(a+)=\hat{f}_{\alpha}(a)$ e acabou.

$(\Longrightarrow)$ Seja $\left(c_{n}\right)_{n \in N^{\prime}}$ uma sequência de elementos de $[a, b]$ tal que $c_{0}=b, c_{n}>c_{n+1}$ e $c_{n} \rightarrow a$.

Seja $\varepsilon>0$.

Portanto, para cada $n>0, \exists \delta_{n}$ gauge de $\left[c_{n}, c_{n-1}\right]$ que é especial relativamente à $c_{n}$ e $c_{n-1}$ tal que $\forall D_{n}=\left(\xi_{i_{n}}, t_{i_{n}}\right) \in T D_{\left[c_{n}, c_{n-1}\right]} \delta_{n}$-fina temos que

$$
\sum_{i_{n}=1}^{\left|D_{n}\right|}\left\|\left[\alpha\left(t_{i_{n}}\right)-\alpha\left(t_{i_{n}-1}\right)\right] f\left(\xi_{i_{n}}\right)-\int_{t_{i_{n}-1}}^{K t_{i_{n}}} d \alpha(t) f(t)\right\|<\frac{\varepsilon}{2^{n}} .
$$


Mas, $\alpha$ é fracamente contínua em $a$ e $\exists \lim _{c ! a}^{K} \int_{c}^{b} d \alpha(t) f(t)=\int_{a}^{k} d \alpha(t) f(t)$, pelo Teorema 2.3.1.

Logo, $\exists n_{0} \in \mathbb{N}$ tal que para $a<c \leq c_{n_{0}}$ temos que

$$
\|[\alpha(c)-\alpha(a)] f(a)\|<\varepsilon
$$

e

$$
\left\|\int_{a}^{K F} d \alpha(t) f(t)\right\|=\left\|\int_{a}^{K{ }^{b}} d \alpha(t) f(t)-\int_{c}^{K^{b}} d \alpha(t) f(t)\right\|<\varepsilon .
$$

Tomemos um gauge $\delta$ de $[a, b]$ tal que $\delta(a)<c_{n_{0}}-a, \delta(\xi) \leq \delta_{n}(\xi)$, se $\xi \in\left[c_{n}, c_{n-1}\right]$ e $\delta$ é especial relativamente à $a$.

Seja $D=\left(\xi_{i}, t_{i}\right) \in T D_{[a, b]} \delta$-fina.

Para cada $n>0$, seja $D_{n}=\left\{\left(\xi_{i}, t_{i}\right) ;\left[t_{i-1}, t_{i}\right] \subset\left[c_{n}, c_{n-1}\right]\right\} \subset D$ e seja também $J_{n}=$ $\left\{\left[t_{i-1}, t_{i}\right] ;\left[t_{i-1}, t_{i}\right] \subset\left[c_{n}, c_{n-1}\right]\right\}$.

Então $D_{n} \in T D_{J_{n}}$ e é $\delta_{n}$-fina, para cada $n>0$.

Seja $D_{n}=\left(\xi_{i_{n}}, t_{i_{n}}\right)$, para cada $n>0$.

Então o LEMA DE SACKS-HENSTOCK (Lema 2.2.3) implica que

$$
\sum_{i_{n}=1}^{\left|D_{J_{n}}\right|}\left\|\left[\alpha\left(t_{i_{n}}\right)-\alpha\left(t_{i_{n}-1}\right)\right] f\left(\xi_{i_{n}}\right)-\int_{t_{i_{n}-1}}^{K t_{i_{n}}} d \alpha(t) f(t)\right\|<\frac{\varepsilon}{2^{n}} .
$$

Portanto,

$$
\begin{gathered}
\sum_{i=1}^{|D|}\left\|\int_{t_{1-1}}^{K t_{1}} d \alpha(t) f(t)-\left[\alpha\left(t_{i}\right)-\alpha\left(t_{i-1}\right)\right] f\left(\xi_{i}\right)\right\| \leq \\
\leq\left\|\int_{a}^{K t_{1}} d \alpha(t) f(t)\right\|+\left\|\left[\alpha\left(t_{1}\right)-\alpha(a)\right] f(a)\right\|+\sum_{i=2}^{|D|}\left\|\int_{t_{i-1}}^{t_{i}} d \alpha(t) f(t)-\left[\alpha\left(t_{i}\right)-\alpha\left(t_{i-1}\right)\right] f\left(\xi_{i}\right)\right\|< \\
<\varepsilon+\varepsilon+\sum_{n=0}^{\infty}\left(\sum_{i_{n}=1}^{\left|D_{n}\right|}\left\|\int_{t_{i_{n}-1}}^{I t_{t_{n}}} d \alpha(t) f(t)-\left[\alpha\left(t_{i_{n}}\right)-\alpha\left(t_{i_{n}-1}\right)\right] f\left(\xi_{i_{n}}\right)\right\|\right)< \\
<2 \varepsilon+\sum_{n=0}^{\infty} \frac{\varepsilon}{2^{n}}<3 \varepsilon .
\end{gathered}
$$


Corolário 1 Se $f \in H([c, b], X), \forall c \in] a, b]$ então $f \in H([a, b], X)$ se e só se $\exists \lim _{c \downarrow a}^{K} \int_{c}^{b} f(t) d t$. Neste caso,

$$
\int_{a}^{k b} f(t) d t=\lim _{c \downarrow a} \int_{c}^{k b} f(t) d t .
$$

Da mesma forma pode-se provar o

Teorema 2.3.4 Sejam $f:[a, b] \rightarrow X$ contínua em a e $\left.\left.\alpha \in H^{f}([c, b], L(X, Y)), \forall c \in\right] a, b\right]$. Então $\alpha \in H^{f}([a, b], L(X, Y))$ se e só se $\exists \lim _{c \downarrow a}{ }^{k} \int_{c}^{b} \alpha(t) d f(t)$. Neste caso,

$$
\int_{a}^{k b} \alpha(t) d f(t)=\lim _{c \downarrow a} \int_{c}^{k b} \alpha(t) d f(t)
$$




\section{REFERÊNCIAS}

[1] R. Henstock, A Riemann-type integral of Lebesgue power, Canad. J. Math., vol. 20 (1968), p. $79-87$.

[2] C. S. Hönig, The abstract Riemann-Stieltjes integral and its applications to Linear Differential Equations with generalized boundary conditions, Notas do Instituto de Matemática e Estatística da Universidade de Sào Paulo, Série Matemática $n^{0} 1,1973$.

[3] C. S. Hönig, The Dirichlet and substitution formulas for Riemann-Stieltjes integrals in Banach spaces, Lecture Notes in Pure and Applied Mathematics, vol 18, Marcel Dekker Inc., 1975, p. $136-189$.

[4] C. S. Hönig, Volterra-Stieltjes integral equations, North Holland Mathematics studies, vol. 16,1975 .

[5] C. S. Hönig, Aplicaçôes da topologia à análise, Projeto Euclides, IMPA-CNPq, 1976.

[6] C. S. Hönig. There is no natural Banach space norm on the space of Kurzweil-HenstockDenjoy-Perron integrable functions. $30^{\circ}$ Seminário Brasileiro de Análise, 1989, p. 387-397.

[7] C. S. Hönig. A riemannian characterization of the Bochner-Lebesgue integral, $35^{0}$ Seminário Brasileiro de Análise, 1992, p. 351-358. 
[8] R. M. McLeod, The generalized Riemann integral, The Carus Math. Monographs, $\mathrm{n}^{0}$ 20,1980 .

[9] E. J. McShane, A unified theory of integration, Americam Math. Monthly, vol. 80 (1973), p. 349-359.

[10] G. C. da Rocha $\mathrm{F}^{0}$, A integral de Riemann vetorial e a geometria de espaços de Banach, Tese de Doutoramento. IME-USP, 1979.

[11] H. L. Royden, Real analysis, Third Edition, Macmillan Comp., 1988. 


\section{ÍNDICE DE SÍMBOLOS}

\begin{tabular}{|c|c|c|c|}
\hline$A C([a, b], X), A C([a, b])$ & 5 & $D_{1} \preceq D_{2}$ & 12 \\
\hline$A_{f}$ & 44 & $D, D^{\bullet}$ & 12 \\
\hline$\alpha(\xi \dot{+}), \alpha(\xi \dot{-})$ & 4 & $|d|$ & $\mathrm{i}$ \\
\hline$\dot{\alpha}_{f}$ & 17,33 & $|D|$ & 12 \\
\hline$B_{\delta}(\xi)$ & ii & $\Delta d$ & $\mathrm{i}$ \\
\hline$B V([a, b], X), B V([a, b])$ & 2 & $\Delta D$ & 12 \\
\hline$B W([a, b], X)$ & 9 & $E([a, b], X)$ & ii \\
\hline $\mathcal{C}([a, b], X)$ & $\mathrm{i}$ & $f(t+), f(t-)$ & ii \\
\hline $\mathcal{C}^{\sigma}([a, b], L(X, Y))$ & ii & $\tilde{f}_{\alpha}$ & 17,33 \\
\hline $\mathcal{C}_{\text {sing }}([a, b])$ & 6 & $F_{\alpha}$ & 44 \\
\hline$D_{[a, b]}$ & $\mathrm{i}$ & $G([a, b], X)$ & 4 \\
\hline$D_{f}$ & $\mathrm{i}$ & $G^{\sigma}([a, b], L(X, Y))$ & 4 \\
\hline$d_{1} \preceq d_{2}$ & $\mathrm{i}$ & $H([a, b], X), H^{\alpha}([a, b], X)$ & 44 \\
\hline
\end{tabular}




\begin{tabular}{|c|c|c|}
\hline$H^{f}([a, b], L(X, Y))$ & 44 & $q s$ \\
\hline$I^{\alpha}([a, b], X)$ & 13 & $R([a, b], X), R^{\alpha}([a, b], X)$ \\
\hline$I^{f}([a, b], L(X, Y))$ & 13 & $R^{f}([a, b], L(X, Y))$ \\
\hline $\int_{a}^{b}, \int_{a}^{b}$ & 13 & $s^{\alpha}(f, D), s^{f}(\alpha, D)$ \\
\hline $\operatorname{Tic}_{a}^{b}$ & 28 & $S V(\alpha), S V_{[a, b]}(\alpha), S V_{d}(\alpha)$ \\
\hline$K([a, b], X), K^{\alpha}([a, b], X)$ & 28 & $S V([a, b], L(X, Y))$ \\
\hline$K^{f}([a, b], L(X, Y))$ & 29 & $T D_{[a, b]}, T D_{[a, b]}^{\bullet}$ \\
\hline$l_{p}([a, b]), L_{p}([a, b])$ & iii & $V(f), V_{[a, b]}(f), V_{d}(f)$ \\
\hline$L(X, Y)$ & $\mathrm{i}$ & $W(\alpha), W_{[a, b]}(\alpha), W_{d}(\alpha)$ \\
\hline $\mathcal{L}_{1}(M)$ & ii & $\omega_{d}(f), \omega_{\delta}(f), \omega_{i}(f)$ \\
\hline${ }^{L} \int_{M}$ & ii & $X^{\prime}$ \\
\hline$m(M)$ & ii & $\chi_{A}$ \\
\hline\|\| & $\mathrm{i}$ & $\left(\xi_{i}, t_{i}\right)$ \\
\hline\|\|$_{p}$ & $\mathrm{iii}$ & $<x, y>$ \\
\hline$|I|$ & ii & \\
\hline
\end{tabular}




\section{ÍNDICE ALFABÉTICO}

divisão interior marcada

divisão marcada

d-oscilação

$\delta$-fina

$\delta$-oscilação

fórmula

de integraçào por partes

de substituiçào

funçào

absolutamente continua

associada

caracteristica

de salto
12

12

i

26

i

$22,37,47$

$22,38,49$

44

ii

† de semivariação limitada

de variação fraca limitada

9

de variação limitada

2

em escada

ii

fracamente continua

ii

fracamente regrada

4

integral

17,33

regrada

4

singular

6

26

26

gauge c-especial

integral

de Bochner-Lebesgue

9

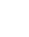


de Riemann-Stieltjes

imprópria

interior

vetorial de Henstock

vetorial de Iiurzweil

vetorial de McShane

vetorial de McShane-Henstock
13

53

13

44

28

iv

variação fraca

semivariação

variação

Teorema de Lebesgue

5

lema

de Cousin

28

de Sacks-Henstock

29,46 


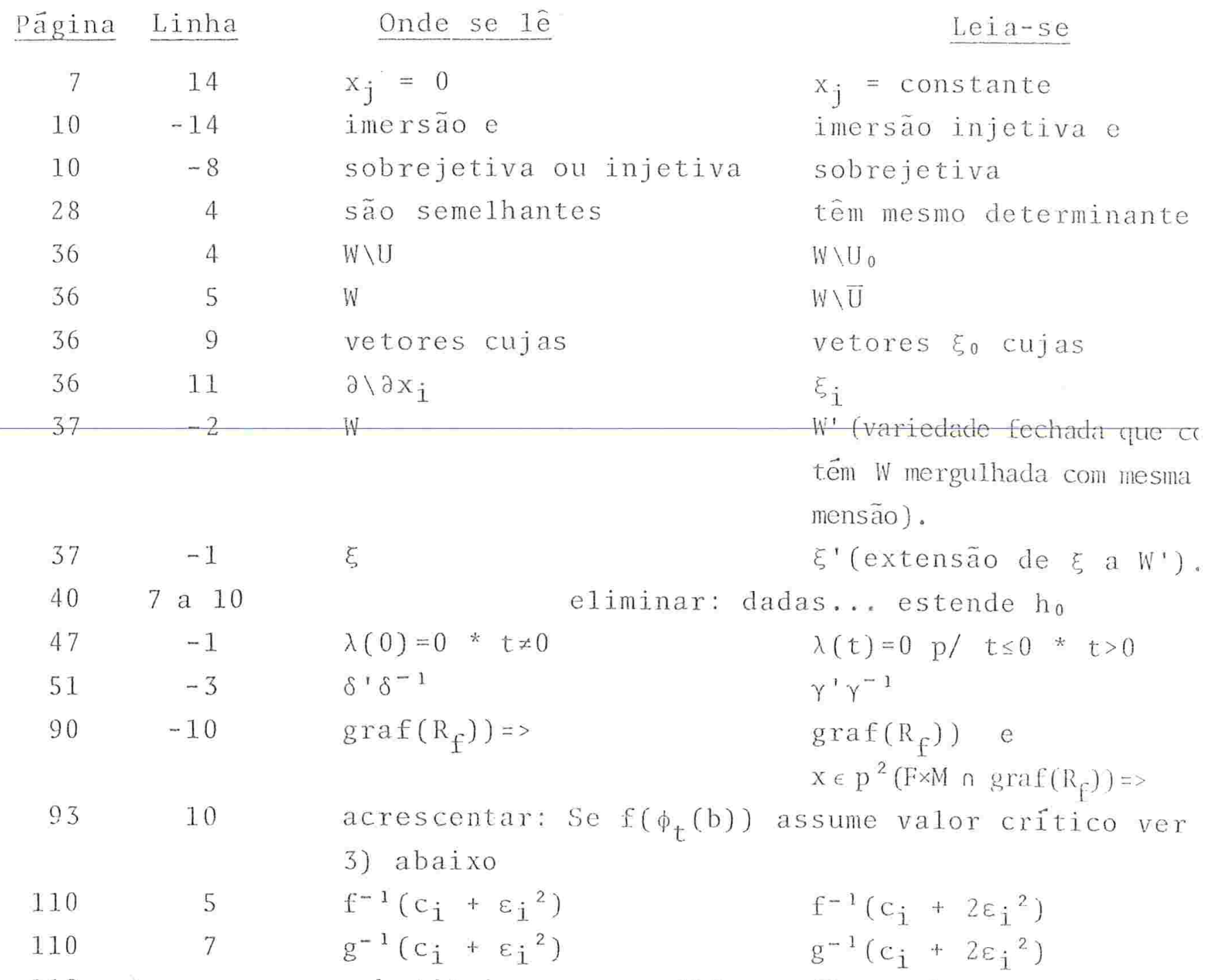

112

substituir por: os difeomorfismos $h_{j}$ podem ser tom dos de modo que existam difeomorfismo

$$
\begin{aligned}
& \alpha_{i}, i+1: B_{i, i+1} \rightarrow B^{\prime} i, i+1 \text { com } g \circ \alpha_{i, j+1}=f \text { e tais que } \\
& \alpha_{i}, i+\left.1\right|_{A_{i} \cap B_{i}, i+1}=h_{i} \text { e }\left.\alpha_{i, i+1}\right|_{B_{i, i+1} \cap A_{i+1}}=h_{i+1} \text {. }
\end{aligned}
$$

De fato, basta considerar campos pseudo-gradientes $\xi$ e $\zeta$ para $f$ e respectivamiente, tais que $\xi(f)=\zeta(g)=1$ fora de uma vizinhança dos pontos críti. cos. Os $h_{j}$ são obtidos por recorrência: dado $h_{i}$, obtém-se $h_{i+1}$ no teorema 0.4 .7 ; partir do difeomorlismo

$$
\psi_{t} n \phi_{-t}: f^{-1}\left(c_{i+1}-2 \varepsilon^{2}{ }_{i+1}\right) \rightarrow g^{-1}\left(c_{i+1}-2 \varepsilon^{2} i+1\right) \text { donde }
$$

$t=c_{i+1}-2 \varepsilon^{2} i+1-c_{i}-2 \varepsilon^{2}$ e $n: f^{-1}\left(c_{i}+2 \varepsilon^{2} i\right) \rightarrow g^{-1}\left(c_{i}+2 \varepsilon^{2}{ }_{i}\right)$ é um difeomor fismo isotöpico com $h_{i} \mid f^{-1}\left(c_{i}+2 \varepsilon^{2}\right)^{2}$ e tal que se $\theta_{1}$ e $\theta_{2}$ são os mergulhos caract rísticos no nível $c_{i+1}-2 \varepsilon^{2}{ }_{i}$ tem-se $n \phi_{-t} \theta_{1}=\psi_{-t} \theta_{2}$.

Definimos $\alpha_{i, i+1}=\psi^{-1} \alpha \phi$ onde, sendo $I=\left[c_{i}+\varepsilon^{2}{ }_{i}, c_{i+1}-\varepsilon^{2}{ }_{i+1}\right]$, $\alpha: f^{-1}\left(c_{i}+2 \varepsilon^{2}{ }_{i}\right) \times I+g^{-1}\left(c_{i}+2 \varepsilon^{2}{ }_{i}\right) \times I$ e uma isotopia entre $h_{i} \mid f^{-1}\left(c_{i}+2 \varepsilon^{2}{ }_{i}\right)$ $n$ tal que

$$
\alpha(x, t)=\left\{\begin{array}{l}
\left(h_{i}(x), t \text { para } t \leq c_{i}+3 \varepsilon^{2} i\right. \\
(n(x), t) \text { para } t \geq c_{i+1}-3 \varepsilon^{2} i+1
\end{array}\right.
$$

0 lema 0.4 .6 mostra que $\left.\alpha_{i, i+1}\right|_{A_{i} \cap B_{i, i+1}}=h_{i}$ e

$$
\left.\alpha_{i, i+1}\right|_{B_{i, i+1} \cap A_{i+1}}=h_{i+1} \text {. }
$$

